\title{
Low-dimensional linear representations of the mapping class group of a nonorientable surface
}

\author{
BŁAŻEJ SZEPIETOWSKI
}

\begin{abstract}
Suppose that $f$ is a homomorphism from the mapping class group $\mathcal{M}\left(N_{g, n}\right)$ of a nonorientable surface of genus $g$ with $n$ boundary components to $\operatorname{GL}(m, \mathbb{C})$. We prove that if $g \geq 5, n \leq 1$ and $m \leq g-2$, then $f$ factors through the abelianization of $\mathcal{M}\left(N_{g, n}\right)$, which is $\mathbb{Z}_{2} \times \mathbb{Z}_{2}$ for $g \in\{5,6\}$ and $\mathbb{Z}_{2}$ for $g \geq 7$. If $g \geq 7, n=0$ and $m=g-1$, then either $f$ has finite image (of order at most two if $g \neq 8$ ), or it is conjugate to one of four "homological representations". As an application we prove that for $g \geq 5$ and $h<g$, every homomorphism $\mathcal{M}\left(N_{g, 0}\right) \rightarrow \mathcal{M}\left(N_{h, 0}\right)$ factors through the abelianization of $\mathcal{M}\left(N_{g, 0}\right)$.
\end{abstract}

20F38; 57N05

\section{Introduction}

For a compact surface $F$, its mapping class group $\mathcal{M}(F)$ is the group of isotopy classes of all, orientation-preserving if $F$ is orientable, homeomorphisms $F \rightarrow F$ equal to the identity on the boundary of $F$. A compact surface of genus $g$ with $n$ boundary components will be denoted by $S_{g, n}$ if it is orientable, and by $N_{g, n}$ if it is nonorientable. If $n=0$ then we drop it in the notation and write simply $S_{g}$ or $N_{g}$. The first integral homology group of $F$ will be denoted by $H_{1}(F)$.

After fixing a basis of $H_{1}\left(S_{g}\right)$, the action of $\mathcal{M}\left(S_{g}\right)$ on $H_{1}\left(S_{g}\right)$ gives rise to a homomorphism $\mathcal{M}\left(S_{g}\right) \rightarrow \operatorname{Sp}(2 g, \mathbb{Z})$, which is well-known to be surjective, and whose kernel is known as the Torelli group. Gluing a disc along each boundary component of $S_{g, n}$ induces an epimorphism $\mathcal{M}\left(S_{g, n}\right) \rightarrow \mathcal{M}\left(S_{g}\right)$, and by composing it with $\mathcal{M}\left(S_{g}\right) \rightarrow \operatorname{Sp}(2 g, \mathbb{Z})$, and then with the inclusion $\operatorname{Sp}(2 g, \mathbb{Z}) \hookrightarrow \operatorname{GL}(2 g, \mathbb{C})$, we obtain the map $\Phi: \mathcal{M}\left(S_{g, n}\right) \rightarrow \mathrm{GL}(2 g, \mathbb{C})$. Recently, the following two results were proved by J Franks, M Handel and M Korkmaz.

Theorem 1.1 (Franks and Handel [8], and Korkmaz [15]) Let $g \geq 2, m \leq 2 g-1$ and let $f: \mathcal{M}\left(S_{g, n}\right) \rightarrow \operatorname{GL}(m, \mathbb{C})$ be a homomorphism. Then $f$ is trivial if $g \geq 3$, and $\operatorname{Im}(f)$ is a quotient of $\mathbb{Z}_{10}$ if $g=2$. 
We say that two homomorphisms $f_{1}, f_{2}$ from a group $G$ to a group $H$ are conjugate if there exits $h \in H$ such that $f_{2}(x)=h f_{1}(x) h^{-1}$ for $x \in G$.

Theorem 1.2 (Korkmaz [16]) For $g \geq 3$, every nontrivial homomorphism

$$
f: \mathcal{M}\left(S_{g, n}\right) \rightarrow \mathrm{GL}(2 g, \mathbb{C})
$$

is conjugate to the map $\Phi$.

In this paper we prove analogous results for $\mathcal{M}\left(N_{g}\right)$. Fix $g \geq 3$. Let $R_{g}$ denote the quotient of $H_{1}\left(N_{g}\right)$ by its torsion. Hence $R_{g}$ is a free $\mathbb{Z}$-module of rank $g-1$. There is a covering $P: S_{g-1} \rightarrow N_{g}$ of degree two. By a theorem of Birman and Chillingworth [3], $\mathcal{M}\left(N_{g}\right)$ is isomorphic to the subgroup of $\mathcal{M}\left(S_{g-1}\right)$ consisting of the isotopy classes of orientation-preserving lifts of homeomorphisms of $N_{g}$, which gives an action of $N_{g}$ on $H_{1}\left(S_{g-1}\right)$. Let $K_{g} \subset H_{1}\left(S_{g-1}\right)$ be the kernel of the composition of the induced map $P_{*}: H_{1}\left(S_{g-1}\right) \rightarrow H_{1}\left(N_{g}\right)$ with the canonical projection $H_{1}\left(N_{g}\right) \rightarrow$ $R_{g}$. Then $K_{g}$ is a $\mathcal{M}\left(N_{g}\right)$-invariant subgroup of rank $g-1$ and we have two homomorphisms

$$
\Psi_{1}: \mathcal{M}\left(N_{g}\right) \rightarrow \operatorname{GL}\left(K_{g}\right) \quad \text { and } \quad \Psi_{2}: \mathcal{M}\left(N_{g}\right) \rightarrow \operatorname{GL}\left(H_{1}\left(S_{g-1}\right) / K_{g}\right),
$$

which after fixing bases will be treated as representations of $\mathcal{M}\left(N_{g}\right)$ in $\operatorname{GL}(g-1, \mathbb{C})$. One may see $\Psi_{1}$ and $\Psi_{2}$ as stemming from the actions of $\mathcal{M}\left(N_{g}\right)$ on homology groups of $N_{g}$ with (local) coefficients in $\mathbb{Z}$ with non-trivial and trivial $\mathbb{Z}\left[\pi_{1}\left(N_{g}\right)\right]$-module structure respectively (see Remark 4.4). We will see that these representations are not conjugate, although $\operatorname{ker} \Psi_{1}=\operatorname{ker} \Psi_{2}$.

Our first result is the following.

Theorem 1.3 Suppose that $n \leq 1, g \geq 5, m \leq g-2$ and $f: \mathcal{M}\left(N_{g, n}\right) \rightarrow \operatorname{GL}(m, \mathbb{C})$ is a nontrivial homomorphism. Then $\operatorname{Im}(f)$ is either $\mathbb{Z}_{2}$ or $\mathbb{Z}_{2} \times \mathbb{Z}_{2}$, the latter case being possible only for $g=5$ or 6 .

Theorem 1.3 was proved in [15], in the more general setting of punctured surfaces, under the additional assumption that $m \leq g-3$ if $g$ is even. Therefore the only novelty of our result is that it also covers the case $m=g-2$ for even $g$. As an application of Theorem 1.3, we prove the following result, which solves Problem 3.3 in [19].

Theorem 1.4 Suppose that $g \geq 5, h<g$ and $f: \mathcal{M}\left(N_{g}\right) \rightarrow \mathcal{M}\left(N_{h}\right)$ is a nontrivial homomorphism. Then $\operatorname{Im}(f)$ is as in Theorem 1.3.

The analogous theorem for mapping class groups of orientable surfaces was proved by Harvey and Korkmaz [13]. In the orientable case the two surfaces need not be closed, as shown in recent theorems of Castel [5], and Aramayona and Souto [1]. 
We will prove that both Theorem 1.3 and Theorem 1.4 fail for $g=4$, by showing that there is a homomorphism from $\mathcal{M}\left(N_{4}\right)$ to $\mathcal{M}\left(N_{3}\right) \cong \mathrm{GL}(2, \mathbb{Z})$ whose image is isomorphic to the infinite dihedral group.

Suppose that $g \geq 7$. Then the abelianization of $\mathcal{M}\left(N_{g}\right)$ is $\mathbb{Z}_{2}$ and we denote by ab: $\mathcal{M}\left(N_{g}\right) \rightarrow \mathbb{Z}_{2}$ the canonical projection. For $i=1,2$ we set $\Psi_{i}^{\prime}=(-1)^{\mathrm{ab}} \Psi_{i}$. Our next result is the following.

Theorem 1.5 Suppose that $g \geq 7, g \neq 8$ and $f: \mathcal{M}\left(N_{g}\right) \rightarrow \operatorname{GL}(g-1, \mathbb{C})$ is a nontrivial homomorphism. Then either $\operatorname{Im}(f) \cong \mathbb{Z}_{2}$, or $f$ is conjugate to one of $\Psi_{1}$, $\Psi_{1}^{\prime}, \Psi_{2}, \Psi_{2}^{\prime}$.

For $g=8$, other representations of $\mathcal{M}\left(N_{8}\right)$ in $\operatorname{GL}(7, \mathbb{C})$ occur, related to the fact that there is an epimorphism $\epsilon: \mathcal{M}\left(N_{8}\right) \rightarrow \operatorname{Sp}\left(6, \mathbb{Z}_{2}\right)$ and the last group admits irreducible representations in $\operatorname{GL}(7, \mathbb{C})$ (see [4]). We prove the following result.

Theorem 1.6 Suppose that $f: \mathcal{M}\left(N_{8}\right) \rightarrow \mathrm{GL}(7, \mathbb{C})$ is a nontrivial homomorphism. Then one of the following holds.

(1) $\operatorname{Im}(f) \cong \mathbb{Z}_{2}$

(2) $f$ or $(-1)^{\mathrm{ab}} f$ factors through $\epsilon: \mathcal{M}\left(N_{8}\right) \rightarrow \operatorname{Sp}\left(6, \mathbb{Z}_{2}\right)$.

(3) $f$ is conjugate to one of $\Psi_{1}, \Psi_{1}^{\prime}, \Psi_{2}, \Psi_{2}^{\prime}$.

To prove our theorems we use the ideas and results from $[8 ; 15 ; 16]$ with necessary modifications. While the case of odd genus is relatively easy, the case of even genus requires much more effort. This phenomenon is typical for the mapping class group of a nonorientable surface.

Throughout this paper we will often have to solve an equation of the form $L=R$, where $L$ and $R$ are products of matrices from $\operatorname{GL}(m, \mathbb{C})$ with some unknown coefficients. Although the dimension $m$ is variable, the calculations of $L$ and $R$ always reduce to multiplication of blocks of size at most $7 \times 7$. With some patience, such calculations could be done by hand, but it is definitely easier to use a computer. We used GAP, but of course, any program that performs symbolic operations on matrices could be used as well.

\section{Notation and algebraic preliminaries}

Suppose that $m \geq 2$ is fixed. We denote by $I_{m}$ the identity matrix of dimension $m$. We will sometimes write simply $I$, if $m$ is clear from the context. We denote by 
$E_{i j}$ the elementary matrix with 1 on the position $(i, j)$ and 0 elsewhere. Suppose that $M_{1}, \ldots, M_{k}$ are nonsingular square matrices of dimensions $m_{1}, \ldots, m_{k}$, where $m_{1}+\cdots+m_{k}=m$. Then we denote by $\operatorname{diag}\left(M_{1}, \ldots, M_{k}\right)$ the $m \times m$ matrix with $M_{1}, \ldots, M_{k}$ on the main diagonal and zeros elsewhere. Set

$$
V=\left(\begin{array}{ll}
1 & 1 \\
0 & 1
\end{array}\right), \quad \hat{V}=\left(\begin{array}{rr}
1 & 0 \\
-1 & 1
\end{array}\right), \quad W=\left(\begin{array}{rrrr}
1 & 1 & 0 & -1 \\
0 & 1 & 0 & 0 \\
0 & -1 & 1 & 1 \\
0 & 0 & 0 & 1
\end{array}\right)
$$

For $2 \leq 2 i \leq m$ we define

$$
A_{i}=\operatorname{diag}\left(I_{2 i-2}, V, I_{m-2 i}\right), \quad B_{i}=\operatorname{diag}\left(I_{2 i-2}, \widehat{V}, I_{m-2 i}\right),
$$

and for $2 \leq 2 j \leq m-2$,

$$
C_{j}=\operatorname{diag}\left(I_{2 j-2}, W, I_{m-2-2 j}\right) .
$$

The proof of the following lemma is straightforward and we leave it as an exercise (cf [16, Lemma 2.2]).

Lemma 2.1 Suppose that $1 \leq k \leq l \leq m / 2$ and $M \in \mathrm{GL}(m, \mathbb{C})$ satisfies $A_{i} M=M A_{i}$, $B_{i} M=M B_{i}$ and $C_{j} M=M C_{j}$ for all $i, j$ such that $k \leq i \leq l, k \leq j \leq l-1$. Then $M$ has the form

$$
\left(\begin{array}{ccc}
* & 0 & * \\
0 & \lambda I_{2(l-k+1)} & 0 \\
* & 0 & *
\end{array}\right)
$$

for some $\lambda \in \mathbb{C}^{*}$, where the top left $\lambda$ of the block $\lambda I_{2(l-k+1)}$ is at the position $(2 k-1,2 k-1)$.

Suppose that $L \in \operatorname{GL}(m, \mathbb{C})$ and $\lambda$ is an eigenvalue of $L$. Then we denote by $\# \lambda$ the multiplicity of $\lambda$. For $k \geq 1$, we denote by $E^{k}(L, \lambda)$ the space $\operatorname{ker}(E-\lambda I)^{k}$. Thus $E^{1}(L, \lambda)$ is the eigenspace of $L$ with respect to $\lambda$, and it will be also denoted by $E(L, \lambda)$. Note that if $L^{\prime} \in \mathrm{GL}(m, \mathbb{C})$ commutes with $L$, then the spaces $E^{k}(L, \lambda)$ are $L^{\prime}$-invariant for $k \geq 1$.

For $k \geq 2$ we denote by $\mathfrak{S}_{k}$ the full symmetric group of the set $\{1, \ldots, k\}$. It is generated by the transpositions $\sigma_{i}=(i, i+1)$ for $1 \leq i \leq k-1$. We will need the following result from the representation theory of the symmetric group; see for example Fulton and Harris [9, Exercise 4.14].

Lemma 2.2 For $k \geq 5, \mathfrak{S}_{k}$ has no irreducible representation (over $\mathbb{C}$ ) of dimension $1<m<k-1$. If $k \geq 7$, then $\mathfrak{S}_{k}$ has two irreducible representations of dimension $k-1:$ the standard one and the tensor product of the standard and sign representations. 


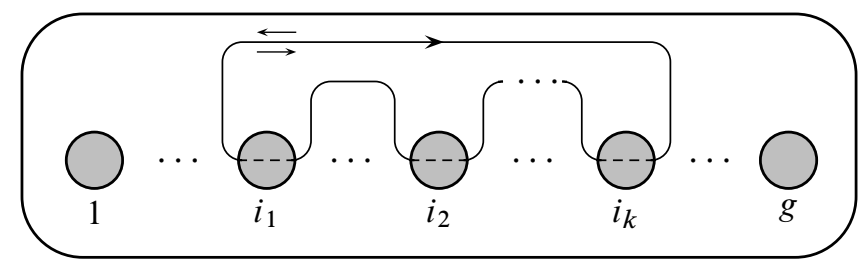

Figure 1: The surface $N_{g, n}$ and the curve $\xi_{I}$ for $I=\left\{i_{1}, i_{2}, \ldots, i_{k}\right\}$
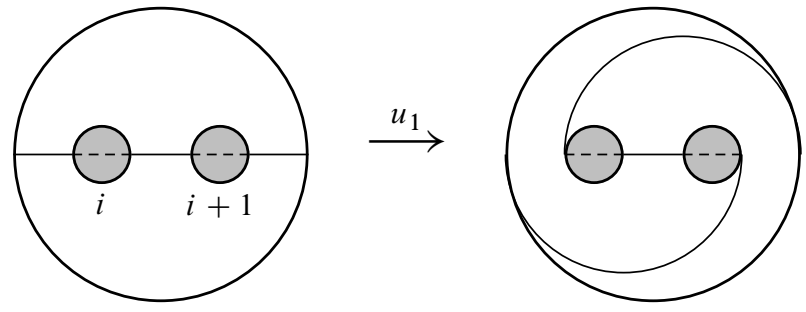

Figure 2: The crosscap transposition $u_{i}$

\section{Mapping class group of a nonorientable surface}

Let $n \in\{0,1\}$ and $g \geq 2$. Let us represent $N_{g, n}$ as a sphere (if $n=0$ ) or a disc (if $n=1)$ with $g$ crosscaps. This means that the interiors of $g$ small pairwise disjoint discs should be removed from the sphere/disc, and then antipodal points in each of the resulting boundary components should be identified. Let us arrange the crosscaps as shown on Figure 1 and number them from 1 to $g$. For each nonempty subset $I \subseteq\{1, \ldots, g\}$ let $\xi_{I}$ be the simple closed curve shown on Figure 1. Note that $\xi_{I}$ is two-sided if and only if $I$ has even number of elements. In this case $t_{\xi_{I}}$ will be the Dehn twist about $\gamma_{I}$ in the direction indicated by arrows on Figure 1.

We will write $\xi_{i}$ instead of $\xi_{\{i\}}$. The following curves will play a special role and so we give them different names:

- $\delta_{i}=\xi_{\{i, i+1\}}$ for $1 \leq i \leq g-1$

- $\varepsilon_{j}=\xi_{\{1,2, \ldots, 2 j\}}$ for $2 \leq 2 j \leq g$

Note that $\varepsilon_{1}=\delta_{1}$.

For $1 \leq i \leq g-1$ we define the crosscap transposition $u_{i}$ to be the isotopy class of the homeomorphism interchanging the $i^{\text {th }}$ and the $(i+1)^{\text {st }}$ crosscaps as shown on Figure 2, and equal to the identity outside a disc containing these crosscaps. 
The groups $\mathcal{M}\left(N_{1, n}\right)$ are trivial for $n \leq 1$ by Epstein [7, Theorem 3.4], and $\mathcal{M}\left(N_{2}\right) \cong$ $\mathbb{Z}_{2} \times \mathbb{Z}_{2}$ by Lickorish [21]. Birman and Chillingworth obtained in [3, Theorem 3] a finite presentation for $\mathcal{M}\left(N_{3}\right)$, from which it is easy to deduce that this group is isomorphic to $\mathrm{GL}(2, \mathbb{Z})$. A direct geometric proof of this fact is given in GonzálezAcuña and Márquez-Bobadilla [12]. For $g \geq 3$, a finite generating set for $\mathcal{M}\left(N_{g, n}\right)$ was given in Chillingworth [6] for $n=0$ and in Stukow [25] for $n>0$. For $n \leq 1$ this set can be reduced to the one given in the following theorem, which can be deduced from the main result of Paris and Szepietowski [23].

Theorem 3.1 For $g \geq 4$ and $n \in\{0,1\}, \mathcal{M}\left(N_{g, n}\right)$ is generated by $u_{g-1}, t_{\varepsilon_{2}}$ and $t_{\delta_{i}}$ for $1 \leq i \leq g-1$.

If $n>1$, then we consider $N_{g, n}$ as the result of gluing $S_{0, n+1}$ to $N_{g, 1}$ along the boundary component. We will need the following relations, satisfied in $\mathcal{M}\left(N_{g, n}\right)$. Those between Dehn twists are the well-known disjointness and braid relations.

(R1) $t_{\delta_{i}} t_{\delta_{j}}=t_{\delta_{j}} t_{\delta_{i}}$ for $|i-j|>1$

(R2) $t_{\varepsilon_{i}} t_{\varepsilon_{j}}=t_{\varepsilon_{j}} t_{\varepsilon_{i}}$ for all $i, j$

(R3) $t_{\varepsilon_{i}} t_{\delta_{j}}=t_{\delta_{j}} t_{\varepsilon_{i}}$ for $j \neq 2 i$

(R4) $t_{\delta_{i}} t_{\delta_{i+1}} t_{\delta_{i}}=t_{\delta_{i+1}} t_{\delta_{i}} t_{\delta_{i+1}}$ for $1 \leq i \leq g-2$

(R5) $t_{\varepsilon_{i}} t_{\delta_{2 i}} t_{\varepsilon_{i}}=t_{\delta_{2 i}} t_{\varepsilon_{i}} t_{\delta_{2 i}}$ for $2 i<g$

The relations involving crosscap transpositions are not so well known and we refer the reader to Paris and Szepietowski [23], and Szepietowski [28], for their proofs.

(R6) $t_{\delta_{i}} u_{j}=u_{j} t_{\delta_{i}}$ for $|i-j|>1$

(R7) $u_{i} u_{j}=u_{j} u_{i}$ for $|i-j|>1$

(R8) $t_{\varepsilon_{i}} u_{j}=u_{j} t_{\varepsilon_{i}}$ for $j>2 i$

(R9) $u_{i} u_{i+1} u_{i}=u_{i+1} u_{i} u_{i+1}$ for $1 \leq i \leq g-2$

(R10) $t_{\delta_{i}} u_{i+1} u_{i}=u_{i+1} u_{i} t_{\delta_{i+1}}$ for $1 \leq i \leq g-2$

(R11) $u_{i+1} t_{\delta_{i}} t_{\delta_{i+1}} u_{i}=t_{\delta_{i}} t_{\delta_{i+1}}$ for $1 \leq i \leq g-2$

(R12) $t_{\delta_{i}} u_{i} t_{\delta_{i}}=u_{i}$ for $1 \leq i \leq g-1$

If follows from (R4) that all $t_{\delta_{i}}$ are conjugate for $1 \leq i \leq g-1$, from (R5) that $t_{\varepsilon_{j}}$ is conjugate to $t_{\delta_{2 j}}$ for $2 j<g$, and from (R12) that $t_{\delta_{i}}$ is conjugate to $t_{\delta_{i}}^{-1}$. Similarly, by (R9) all $u_{i}$ are conjugate for $1 \leq i \leq g-1$, and by (R11) $u_{i}$ is conjugate to $u_{i}^{-1}$.

For a group $G$ we denote the abelianization $G /[G, G]$ by $G^{\mathrm{ab}}$. The following theorem is proved in Korkmaz [17] for $n=0$ and generalised to $n>0$ in Stukow [25]. 
Theorem 3.2 For $n \leq 1$ and $g \geq 3, \mathcal{M}\left(N_{g, n}\right)^{\mathrm{ab}}$ has the following presentation as a $\mathbb{Z}$-module:

$$
\begin{aligned}
\left\langle\left[t_{\delta_{1}}\right],\left[t_{\varepsilon_{2}}\right],\left[u_{1}\right]\right| 2\left[t_{\delta_{1}}\right] & \left.=2\left[t_{\varepsilon_{2}}\right]=2\left[u_{1}\right]=0\right\rangle & & \text { if } g=4 \\
\left\langle\left[t_{\delta_{1}}\right],\left[u_{1}\right]\right| 2\left[t_{\delta_{1}}\right] & \left.=2\left[u_{1}\right]=0\right\rangle & & \text { if } g \in\{3,5,6\} \\
\left\langle\left[u_{1}\right]\right| 2\left[u_{1}\right] & =0\rangle & & \text { if } g \geq 7
\end{aligned}
$$

In particular, for $g \geq 7$ we have $\left[t_{\delta_{1}}\right]=0$.

Lemma 3.3 For $g \geq 5$ and $n \leq 1$, let $\alpha, \beta$ be two-sided curves on $N_{g, n}$, intersecting transversally in one point. If $f: \mathcal{M}\left(N_{g, n}\right) \rightarrow G$ is a homomorphism, such that $f\left(t_{\alpha}\right)$ commutes with $f\left(t_{\beta}\right)$, then $\operatorname{Im}(f)$ is abelian.

Proof Let $N=N_{g, n}$ and $\mathcal{M}=\mathcal{M}\left(N_{g, n}\right)$. Fix a regular neighbourhood $A$ of $\alpha \cup \beta$. Note that $A$ is homeomorphic to $S_{1,1}$ and $N \backslash A$ is homeomorphic to $N_{g-2,1}$. It follows that for each $i \leq g-2$ there is a homeomorphism $h: N \rightarrow N$ such that $h(\alpha)=\delta_{i}$ and $h(\beta)=\delta_{i+1}$. It follows that $h t_{\alpha} h^{-1}=t_{\delta_{i}}^{\varepsilon_{1}}$ and $h t_{\beta} h^{-1}=t_{\delta_{i+1}}^{\varepsilon_{2}}$, where $\varepsilon_{j} \in\{-1,1\}$ for $j=1,2$. Hence $f\left(t_{\delta_{i}}\right)$ commutes with $f\left(t_{\delta_{i+1}}\right)$, and by the braid relation (R4), $f\left(t_{\delta_{i}}\right)=f\left(t_{\delta_{i+1}}\right)$. Analogously, $f\left(t_{\varepsilon_{2}}\right)=f\left(t_{\delta_{4}}\right)$. By Theorem 3.1, $\operatorname{Im}(f)$ is generated by $f\left(t_{\delta_{1}}\right)$ and $f\left(u_{g-1}\right)$, and since $u_{g-1}$ commutes with $t_{\delta_{1}}$, $\operatorname{Im}(f)$ is abelian.

Lemma 3.4 Suppose that $g \geq 4$ and $f: \mathcal{M}\left(N_{g, n}\right) \rightarrow G$ is a homomorphism. If $f\left(t_{\varepsilon_{i}}\right)=f\left(t_{\delta_{j}}\right)$ for some $2 i+1 \leq j \leq g-1$, then $f\left(t_{\delta_{1}}^{2}\right)=1$.

Proof Set $x=f\left(t_{\varepsilon_{i}}\right)=f\left(t_{\delta_{j}}\right)$ and $y=f\left(u_{j}\right)$. By the relation (R8) we have $x y=y x$, and by (R12), $x y x=y$. Hence $x^{2}=1$, which finishes the proof, because $t_{\delta_{j}}$ is conjugate to $t_{\delta_{1}}$.

Let $g=2 r+s$, where $r \geq 1, s \in\{1,2\}$ and $S=S_{g-1}$. Consider $S$ as being embedded in $\mathbb{R}^{3}$ in such a way that it is invariant under the reflections about the $x y$, $x z$ and $y z$ planes, as shown on Figure 3. We define a homeomorphism $j: S \rightarrow S$ as $j(x, y, z)=(-x,-y,-z)$. The quotient space $S / j$ is a nonorientable surface of genus $g$ and the projection $p: S \rightarrow S / j$ is a covering map of degree 2 . Let $S^{\prime}$ be the subsurface of $S$ consisting of points $(x, y, z) \in S$ with $x \leq-\varepsilon$, where $\varepsilon$ is a positive constant, so small that $S^{\prime}$ is homeomorphic to $S_{r, s}$. If $g$ is even, then one of the boundary components of $S^{\prime}$ is isotopic to $\alpha_{r+1}$. In this paper we identify isotopic curves, and therefore we will treat $\alpha_{r+1}$ as a curve on $S^{\prime}$. Note that the restriction of $p$ to $S^{\prime}$ is an embedding. For odd $g$ we define $\gamma^{\prime}$ to be the arc of $\gamma_{r}$ consisting of points with $x \leq 0$. For even $g$ we define $\beta^{\prime}$ to be the arc of $\beta_{r+1}$ consisting of points with $x \leq 0$. Note that $p\left(\gamma^{\prime}\right)$ and $p\left(\beta^{\prime}\right)$ are one-sided simple closed curves on $S / j$. 

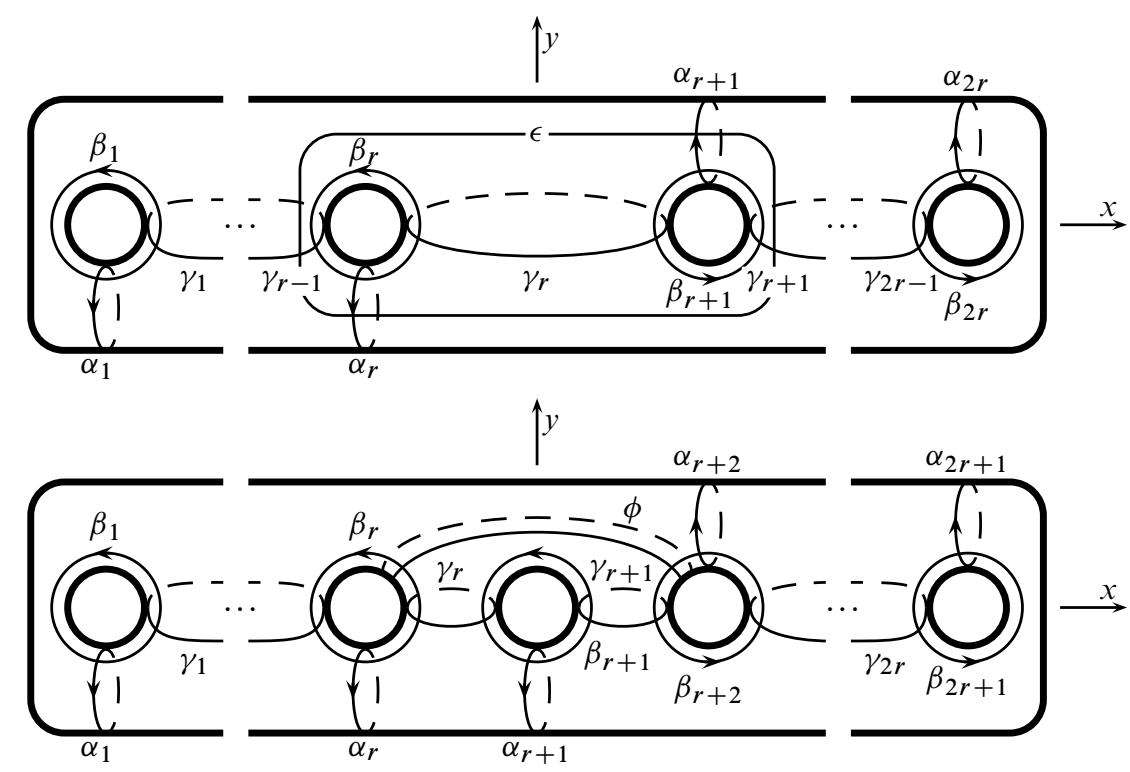

Figure 3: The surface $S_{g-1}$ for $g=2 r+1$ (top) and $g=2 r+2$ (bottom)

Proposition 3.5 There is a homeomorphism $\varphi: S_{g-1} / j \rightarrow N_{g}$ such that, for $P=$ $\varphi \circ p$, up to isotopy:
(1) $P\left(\beta_{i}\right)=\delta_{2 i}$ for $1 \leq i \leq r$
(2) $P\left(\alpha_{i}\right)=\varepsilon_{i}$ for $2 \leq 2 i \leq g$
(3) $P\left(\gamma_{i}\right)=\delta_{2 i+1}$ for $2 \leq 2 i \leq g-2$
(4) $P\left(\gamma^{\prime}\right)=\xi_{g}$ if $g$ is odd
(5) $P\left(\beta^{\prime}\right)=\xi_{g}$ if $g$ is even

Proof By altering the curves $\delta_{i}, \varepsilon_{j}$ and $\xi_{g}$ by a small isotopy, we may assume that they intersect each other minimally. The curves $\delta_{i}$ for $1 \leq i \leq g-1$ form a chain of two-sided curves, which means that $\delta_{i}$ and $\delta_{j}$ intersect at one point if $|i-j|=1$, and they are disjoint otherwise. The one-sided curve $\xi_{g}$ intersects $\delta_{g-1}$ at one point and is disjoint from $\delta_{i}$ for $i<g-1$. Let $\Sigma$ be a regular neighbourhood of the union of $\delta_{i}$ for $1 \leq i \leq g-$ 1 , and let $M$ be the union of $\Sigma$ with a regular neighbourhood of $\xi_{g}$. Observe that $\Sigma$ and $M$ are homeomorphic to $S_{r, s}$ and $N_{g, 1}$ respectively. We may choose $\Sigma$ big enough to contain the curves $\varepsilon_{i}$ for $2 \leq 2 i \leq g$ (if $g$ is even, then one of the boundary components of $\Sigma$ is isotopic to $\left.\varepsilon_{r+1}\right)$. Let $M^{\prime} \subset S_{g-1} / j$ be the union of $p\left(S^{\prime}\right)$ and a regular neighbourhood of the one-sided curve $p\left(\gamma^{\prime}\right)$ if $g$ is odd, or $p\left(\beta^{\prime}\right)$ if $g$ is even. There is a homeomorphism $\varphi: M^{\prime} \rightarrow M$ such that for $P=\varphi \circ p$ we have $P\left(S^{\prime}\right)=\Sigma$ and 
$P$ maps the chain $\left(\alpha_{1}, \beta_{1}, \gamma_{1}, \beta_{2}, \gamma_{2}, \ldots, \beta_{r}, \gamma^{\prime}\right)\left(\right.$ or $\left.\left(\alpha_{1}, \beta_{1}, \gamma_{1}, \beta_{2}, \gamma_{2}, \ldots, \gamma_{r}, \beta^{\prime}\right)\right)$ on $\left(\delta_{1}, \ldots, \delta_{g-1}, \xi_{g}\right)$. Such a $P$ satisfies conditions (1), (3), (4), (5) of the lemma. Since $\varepsilon_{i}, \varepsilon_{i+1}$ and $\delta_{2 i+1}$ bound a pair of pants in $\Sigma$ for $2 \leq 2 i \leq g-2, \varphi$ may be taken to also satisfy (2). Finally, since $\left(S_{g-1} / j\right) \backslash M^{\prime}$ and $N_{g} \backslash M$ are discs, $\varphi$ may be extended to $\varphi: S_{g-1} / j \rightarrow N_{g}$.

Corollary 3.6 There is a homomorphism $\iota: \mathcal{M}\left(S^{\prime}\right) \rightarrow \mathcal{M}\left(N_{g, n}\right)$ such that:

- $\iota\left(t_{\beta_{i}}\right)=t_{\delta_{2 i}}$ for $1 \leq i \leq r$

- $\iota\left(t_{\alpha_{i}}\right)=t_{\varepsilon_{i}}$ for $2 \leq 2 i \leq g$

- $\iota\left(t_{\gamma_{i}}\right)=t_{\delta_{2 i+1}}$ for $2 \leq 2 i \leq g-2$

Here the Dehn twists about the curves on $S^{\prime}$ are right-handed with respect to the standard orientation.

Proof By the proof of Proposition 3.5, the restriction of $P$ to $S^{\prime}$ is a homeomorphism onto $\Sigma$ satisfying conditions (1), (2), (3). There is an induced isomorphism $\mathcal{M}\left(S^{\prime}\right) \rightarrow$ $\mathcal{M}(\Sigma)$, which may be composed with the homomorphism $\mathcal{M}(\Sigma) \rightarrow \mathcal{M}\left(N_{g, n}\right)$ induced by the inclusion $\Sigma \hookrightarrow N_{g, n}$, for any $n \geq 0$, to obtain $\iota$.

For any homeomorphism $h: N_{g} \rightarrow N_{g}$ there is a unique orientation preserving lift $\tilde{h}: S_{g-1} \rightarrow S_{g-1}$ such that $h \circ P=P \circ \tilde{h}$. By [3], the mapping $h \mapsto \tilde{h}$ induces a monomorphism $\theta: \mathcal{M}\left(N_{g}\right) \rightarrow \mathcal{M}\left(S_{g-1}\right)$. The following proposition follows from [3] and [28, Theorem 10], where the lift of a crosscap transposition is determined.

Proposition 3.7 There is a monomorphism $\theta: \mathcal{M}\left(N_{g}\right) \rightarrow \mathcal{M}\left(S_{g-1}\right)$ such that

$$
\theta\left(t_{\varepsilon_{i}}\right)=t_{\alpha_{i}} t_{\alpha_{g-i}}^{-1}, \quad \theta\left(t_{\delta_{2 i}}\right)=t_{\beta_{i}} t_{\beta_{g-i}}^{-1}, \quad \theta\left(t_{\delta_{2 j+1}}\right)=t_{\gamma_{j}} t_{\gamma_{g-1-j}}^{-1},
$$

for $1 \leq i \leq r, 2 \leq 2 j \leq g-2$ and

$$
\theta\left(u_{g-1}\right)= \begin{cases}{ }_{\beta_{r}}^{-1} t_{\beta_{r+1}}\left(t_{\gamma_{r}} t_{\beta_{r}} t_{\beta_{r+1}}\right)^{2} t_{\epsilon}^{-1} & \text { if } g=2 r+1, \\ t_{\gamma_{r}}^{-1} t_{\gamma_{r+1}}\left(t_{\beta_{r+1}} t_{\gamma_{r}} t_{\gamma_{r+1}}\right)^{2} t_{\phi}^{-1} & \text { if } g=2 r+2 .\end{cases}
$$

\section{Homological representations}

Fix $g \geq 3$ and let $S=S_{g-1}, N=N_{g}$ and $P: S \rightarrow N$ be as in the previous section. The group $H_{1}(S)$ is a free $\mathbb{Z}$-module of rank $2(g-1)$ and the homology classes $a_{i}=\left[\alpha_{i}\right], b_{i}=\left[\beta_{i}\right]$ for $1 \leq i \leq g-1$ form its basis, which is a symplectic basis with respect to the algebraic intersection form:

$$
\left\langle a_{i}, a_{j}\right\rangle=0, \quad\left\langle b_{i}, b_{j}\right\rangle=0, \quad\left\langle a_{i}, b_{j}\right\rangle=\delta_{i j}
$$


Let $\Phi: \mathcal{M}(S) \rightarrow \operatorname{Sp}\left(H_{1}(S)\right)$ be the homomorphism induced by the action of $\mathcal{M}(S)$ on $H_{1}(S)$. If $\gamma$ is an oriented simple closed curve on $S,[\gamma] \in H_{1}(S)$ is its homology class, and $t_{\gamma}$ is the right Dehn twist, then $\Phi\left(t_{\gamma}\right)$ is the transvection

$$
\Phi\left(t_{\gamma}\right)(h)=h+\langle[\gamma], h\rangle[\gamma] \text { for } h \in H_{1}(S) .
$$

From (1) we immediately obtain that, with respect to the basis $\left(a_{1}, b_{1}, \ldots, a_{g-1}, b_{g-1}\right)$, we have

$$
\Phi\left(t_{\alpha_{i}}\right)=A_{i}, \quad \Phi\left(t_{\beta_{i}}\right)=B_{i}, \quad \Phi\left(t_{\gamma_{j}}\right)=C_{j},
$$

for $1 \leq i \leq g-1,1 \leq j \leq g-2$, where $A_{i}, B_{i}$ and $C_{j}$ are the matrices defined in Section 2 .

The group $H_{1}(N)$ has the following presentation, as a $\mathbb{Z}$-module:

$$
H_{1}(N)=\left\langle x_{1}, \ldots, x_{g} \mid 2\left(x_{1}+\cdots+x_{g}\right)=0\right\rangle,
$$

where $x_{i}=\left[\xi_{i}\right]$. Set $k=x_{1}+\cdots+x_{g}$ and $R=H_{1}(N) /\langle k\rangle$. Observe that $k$ is the unique element of order two in $H_{1}(N)$ and $R$ is a free $\mathbb{Z}$-module of rank $g-1$.

The map $P: S \rightarrow N$ induces $P_{*}: H_{1}(S) \rightarrow H_{1}(N)$, such that, for $1 \leq i \leq r$,

$$
\begin{aligned}
& P_{*}\left(a_{i}\right)=x_{1}+\cdots+x_{2 i}=-P_{*}\left(a_{g-i}\right), \\
& P_{*}\left(b_{i}\right)=x_{2 i}+x_{2 i+1}=P_{*}\left(b_{g-i}\right),
\end{aligned}
$$

and if $g=2 r+2$, then

$$
P_{*}\left(a_{r+1}\right)=x_{1}+\cdots+x_{g}=k, \quad P_{*}\left(b_{r+1}\right)=2 x_{g} .
$$

Let $q: H_{1}(S) \rightarrow R$ be the composition of $P_{*}$ with the canonical projection $H_{1}(N) \rightarrow$ $R$, and set $K=\operatorname{ker} q$. Observe that $K$ has rank $g-1$ and the following elements form its basis:

$$
\begin{aligned}
e_{i}=a_{i}+a_{g-i}, \quad e_{r+i}=b_{i}-b_{g-i} & \text { for } 1 \leq i \leq r \\
e_{2 r+1}=a_{r+1} & \text { for } g=2 r+2
\end{aligned}
$$

We also set

$$
\begin{aligned}
f_{i}=b_{i}, \quad f_{r+i}=a_{g-i} & \text { for } 1 \leq i \leq r, \\
f_{2 r+1}=b_{r+1} & \text { for } g=2 r+2 .
\end{aligned}
$$

The elements $e_{i}, f_{i}$ for $1 \leq i \leq g-1$ form a symplectic basis of $H_{1}(S)$. It follows that $H_{1}(S) / K$ is a free $\mathbb{Z}$-module of rank $g-1$ that is canonically isomorphic to $R$ if $g$ is odd, or to an index-two subgroup of $R$ if $g$ is even. The group $\mathcal{M}(N)$ 
acts on $H_{1}(S)$ by the composition $\Phi \circ \theta: \mathcal{M}(N) \rightarrow \operatorname{Sp}\left(H_{1}(S)\right)$. Observe that $K$ is $M(N)$-invariant and hence we have two $(g-1)$-dimensional representations

$$
\Psi_{1}: \mathcal{M}(N) \rightarrow \mathrm{GL}(K), \quad \Psi_{2}: \mathcal{M}(N) \rightarrow \mathrm{GL}\left(H_{1}(S) / K\right) .
$$

Lemma 4.1 $\operatorname{ker} \Psi_{1}=\operatorname{ker} \Psi_{2}$ and $\theta\left(\operatorname{ker} \Psi_{1}\right) \subset \operatorname{ker} \Phi$.

Proof Fix the basis $\left(e_{1}, \ldots, e_{g-1}, f_{1}, \ldots, f_{g-1}\right)$ of $H_{1}(S)$. For any $x \in \mathcal{M}(N)$ let $X$ be the matrix of $\Phi(\theta(x))$. Since $\Phi(\theta(x))$ preserves $K$, we have

$$
X=\left(\begin{array}{cc}
X_{1} & Y \\
0 & X_{2}
\end{array}\right)
$$

where $X_{1}, X_{2}, Y$ are $(g-1) \times(g-1)$ matrices. Furthermore, $X_{1}$ is the matrix of $\Psi_{1}(x)$ with respect to the basis $\left(e_{i}\right)_{1 \leq i \leq g-1}$ of $K$, and $X_{2}$ is the matrix of $\Psi_{2}(x)$ with respect to the basis $\left(f_{i}+K\right)_{1 \leq i \leq g-1}$ of $H_{1}(S) / K$. The algebraic intersection form on $H_{1}(S)$ has the matrix

$$
\Omega=\left(\begin{array}{cc}
0 & I_{g-1} \\
-I_{g-1} & 0
\end{array}\right)
$$

Since $X$ is symplectic, we have $X^{t} \Omega X=\Omega$, which gives $X_{1}^{t} X_{2}=I$. Therefore $X_{1}=I \Leftrightarrow X_{2}=I$, which proves $\operatorname{ker} \Psi_{1}=\operatorname{ker} \Psi_{2}$.

To prove the second assertion, suppose that $x \in \operatorname{ker} \Psi_{1}$. Then

$$
X=\left(\begin{array}{cc}
I_{g-1} & Y \\
0 & I_{g-1}
\end{array}\right)
$$

and we have to show $Y=0$. Let $j_{*}: H_{1}(S) \rightarrow H_{1}(S)$ be the map induced by the covering involution $j$. We have $j_{*}\left(a_{i}\right)=-a_{g-i}$ and $j_{*}\left(b_{i}\right)=b_{g-i}$ for $1 \leq i \leq g-1$. It follows that the matrix of $j_{*}$ with respect to the basis $\left(e_{1}, \ldots, e_{g-1}, f_{1}, \ldots, f_{g-1}\right)$ has the form

$$
J=\left(\begin{array}{cc}
-I_{g-1} & T \\
0 & I_{g-1}
\end{array}\right)
$$

for some $T$. Since $\theta(x)$ commutes with $j$, we have $X J=J X$, which gives $Y+T=$ $-Y+T$, hence $Y=0$.

Note that $\operatorname{ker} \Phi$ is the Torelli group, which is well known to be torsion-free, and since $\theta$ is a monomorphism, we immediately obtain the following.

Corollary 4.2 $\operatorname{ker} \Psi_{1}$ is torsion-free. 
Remark 4.3 Let $H$ denote the subgroup of $\mathcal{M}(N)$ consisting of the elements inducing the identity on $H_{1}(N)$. It was proved in Gastesi [11] that $\theta(H) \subset \operatorname{ker} \Phi$. We leave it as an exercise to check that if $g$ is odd, then $H=\operatorname{ker} \Psi_{2}$, whereas if $g$ is even, then $H$ is an index-two subgroup of $\operatorname{ker} \Psi_{2}$. In the latter case, if $g=2 r+2$, then we have $\operatorname{ker} \Psi_{2}=H \cup t_{\varepsilon_{r+1}} H$.

Remark 4.4 There is a nontrivial action of $\pi_{1}(N)$ on $\mathbb{Z}$ defined as follows: $\gamma \in$ $\pi_{1}(N)$ acts by multiplication by 1 or -1 according to whether $\gamma$ preserves or reverses local orientations of $N$. This action gives rise to homology groups with local coefficients $H_{*}(N, \widetilde{\mathbb{Z}})$, where $\widetilde{\mathbb{Z}}$ is $\mathbb{Z}$ with the nontrivial $\mathbb{Z}\left[\pi_{1}(N)\right]$-module structure. By Hatcher [14, Example 3H.3], we have the exact sequence

$$
H_{2}(N) \rightarrow H_{1}(N, \widetilde{\mathbb{Z}}) \rightarrow H_{1}(S) \stackrel{P_{*}}{\rightarrow} H_{1}(N),
$$

which is a part of a long exact sequence of homology groups. Since $\mathrm{H}_{2}(\mathrm{~N})=0$, we have a $\mathcal{M}(N)$-equivariant isomorphism $H_{1}(N, \widetilde{\mathbb{Z}}) \cong \operatorname{ker} P_{*}$. If $g$ is odd, then ker $P_{*}=K$, whereas if $g$ is even, then $\operatorname{ker} P_{*}$ is an index-two subgroup of $K$. Therefore the representations $\Psi_{1}$ and $\Psi_{2}$ may be seen as coming from the actions of $\mathcal{M}(N)$ on $H_{1}(N, \widetilde{\mathbb{Z}})$ and $H_{1}(N)$ respectively.

For $K$ we fix the basis

$$
\begin{aligned}
& \left(e_{1}, e_{r+1}, \ldots, e_{r}, e_{2 r}\right) \quad \text { if } g=2 r+1, \\
& \left(e_{1}, e_{r+1}, \ldots, e_{r}, e_{2 r}, e_{2 r+1}\right) \quad \text { if } g=2 r+2 .
\end{aligned}
$$

For $H_{1}(S) / K$ we fix the basis

$$
\begin{aligned}
& \left(a_{1}+K, b_{1}+K, \ldots, a_{r}+K, b_{r}+K\right) \quad \text { if } g=2 r+1, \\
& \left(a_{1}+K, b_{1}+K, \ldots, a_{r}+K, b_{r}+K, b_{r+1}+K\right) \quad \text { if } g=2 r+2 .
\end{aligned}
$$

Having fixed bases for $K$ and $H_{1}(S) / K$ we can now compute, for $\Psi_{1}$ and $\Psi_{2}$, the images of the generators of $\mathcal{M}(N)$. This is done by a straightforward calculation, using Proposition 3.7 and the formula (1). For $k=1,2$ and $1 \leq i \leq r, 1 \leq j \leq r-1$, we have

$$
\Psi_{k}\left(t_{\varepsilon_{i}}\right)=A_{i}, \quad \Psi_{k}\left(t_{\delta_{2 i}}\right)=B_{i}, \quad \Psi_{k}\left(t_{\delta_{2 j+1}}\right)=C_{j} .
$$

If $g=2 r+1$, then

$$
\Psi_{1}\left(u_{g-1}\right)=\left(\begin{array}{ccc}
I_{g-3} & 0 & 0 \\
0 & 1 & 0 \\
0 & 1 & -1
\end{array}\right), \quad \Psi_{2}\left(u_{g-1}\right)=\left(\begin{array}{crr}
I_{g-3} & 0 & 0 \\
0 & -1 & 0 \\
0 & -1 & 1
\end{array}\right) .
$$


If $g=2 r+2$, then

$$
\begin{array}{ll}
\Psi_{1}\left(t_{\delta_{g-1}}\right)=\left(\begin{array}{cccc}
I_{g-4} & 0 & 0 & 0 \\
0 & 1 & 1 & 0 \\
0 & 0 & 1 & 0 \\
0 & 0 & -2 & 1
\end{array}\right), & \Psi_{2}\left(t_{\delta_{g-1}}\right)=\left(\begin{array}{rrrrr}
I_{g-4} & 0 & 0 & 0 \\
0 & 1 & 1 & -2 \\
0 & 0 & 1 & 0 \\
0 & 0 & 0 & 1
\end{array}\right), \\
\Psi_{1}\left(u_{g-1}\right)=\left(\begin{array}{rrrr}
I_{g-4} & 0 & 0 & 0 \\
0 & 1 & -1 & 1 \\
0 & 0 & 1 & 0 \\
0 & 0 & 2 & -1
\end{array}\right), & \Psi_{2}\left(u_{g-1}\right)=\left(\begin{array}{rrrr}
I_{g-4} & 0 & 0 & 0 \\
0 & 1 & 1 & -2 \\
0 & 0 & 1 & 0 \\
0 & 0 & 1 & -1
\end{array}\right) .
\end{array}
$$

It is easy to see that $\Psi_{1}$ and $\Psi_{2}$ are not conjugate as homomorphisms to $\operatorname{GL}(g-1, \mathbb{C})$. For, suppose that there is $M \in \mathrm{GL}(g-1, \mathbb{C})$ such that $\Psi_{1}(x)=M \Psi_{2}(x) M^{-1}$ for all $x \in \mathcal{M}(N)$. Then $M$ commutes with $A_{i}, B_{i}, C_{j}$ for $1 \leq i \leq r, 1 \leq j \leq r-1$, and by Lemma 2.1, $M=\alpha I_{2 r}$ if $g=2 r+1$, or $M=\operatorname{diag}\left(\alpha I_{2 r}, \beta\right)$ if $g=2 r+2$, for $\alpha, \beta \in \mathbb{C}$. In either case it is impossible that $\Psi_{1}\left(u_{g-1}\right)=M \Psi_{2}\left(u_{g-1}\right) M^{-1}$.

\section{Homomorphisms from $\mathcal{M}\left(N_{g, n}\right)$ to $\operatorname{GL}(m, \mathbb{C})$ for $m<g-1$}

The aim of this section is to prove Theorem 1.3. The proof is divided in two parts.

Proof of Theorem 1.3 for $(\boldsymbol{g}, \boldsymbol{m}) \neq(\mathbf{6}, \mathbf{4})$ Suppose that $n \in\{0,1\}, g=2 r+s$ for $r \geq 2, s \in\{1,2\}, m \leq g-2$ and $f: \mathcal{M}\left(N_{g, n}\right) \rightarrow \mathrm{GL}(m, \mathbb{C})$ is a homomorphism. By Theorem 3.2, it suffices to prove that $\operatorname{Im}(f)$ is abelian. Let $S^{\prime}=S_{r, s}$ and $\iota: \mathcal{M}\left(S^{\prime}\right) \rightarrow$ $\mathcal{M}\left(N_{g, n}\right)$ be the homomorphism from Corollary 3.6. Set $f^{\prime}=f \circ \iota$ and observe that if $\operatorname{Im}\left(f^{\prime}\right)$ is abelian, then so is $\operatorname{Im}(f)$, by Lemma 3.3.

Suppose that $m \leq 2 r-1$. Then $\operatorname{Im}\left(f^{\prime}\right)$ is either trivial or cyclic by Theorem 1.1 and we are done. This finishes the proof for odd $g$.

Suppose that $g=2 r+2$ for $r \geq 3$ and $m=2 r$. By Theorem 1.2, $f^{\prime}$ is either trivial or conjugate to the homological representation $\Phi$. In the former case we are done. In the latter case, by the definition of $\Phi$ we have $\Phi\left(t_{\gamma_{r}}\right)=\Phi\left(t_{\alpha_{r}}\right)$ because the curves $\gamma_{r}$ and $\alpha_{r}$ become isotopic after gluing discs to the boundary of $S^{\prime}$. It follows that $f\left(t_{\delta_{2 r+1}}\right)=f\left(t_{\varepsilon_{r}}\right)$ and, by Lemma 3.4, $f\left(t_{\delta_{1}}^{2}\right)=1$. This is a contradiction because $\Phi\left(t_{\alpha_{1}}\right)$ has infinite order.

In order to prove Theorem 1.3 for $(g, m)=(6,4)$, we first prove some lemmas.

Lemma 5.1 Suppose that $n \leq 1$ and $f: \mathcal{M}\left(N_{6, n}\right) \rightarrow \operatorname{GL}(4, \mathbb{C})$ is a homomorphism such that $f\left(t_{\delta_{1}}^{2}\right)=1$. Then $\operatorname{Im}(f)$ is abelian. 
Proof Let $H$ be the normal closure of $t_{\delta_{1}}^{2}$ in $\mathcal{M}\left(N_{6, n}\right)$ and set $G=\mathcal{M}\left(N_{6, n}\right) / H$. We have an induced homomorphism $f^{\prime}: G \rightarrow \operatorname{GL}(4, \mathbb{C})$ such that $f=f^{\prime} \circ \pi$, where $\pi: \mathcal{M}\left(N_{6, n}\right) \rightarrow G$ is the canonical projection. By the relations (R1), (R4), the mapping $\rho\left(\sigma_{i}\right)=\pi\left(t_{\delta_{i}}\right)$, where $\sigma_{i}$ is the transposition $(i, i+1)$ for $1 \leq i \leq 5$, defines a homomorphism $\rho: \mathfrak{S}_{6} \rightarrow G$. Let $\phi: \mathfrak{S}_{6} \rightarrow \mathrm{GL}(4, \mathbb{C})$ be the composition $f^{\prime} \circ \rho$. By Lemma 2.2, $\phi$ is the direct sum of one-dimensional representations. In particular the image of $\phi$ is abelian, and so is $\operatorname{Im}(f)$ by Lemma 3.3.

Let $R$ be the subsurface obtained by removing from $N_{6, n}$ a regular neighbourhood of $\delta_{1} \cup \delta_{2}$. Note that $R$ is homeomorphic to $N_{4, n+1}$. The homomorphism $\mathcal{M}(R) \rightarrow$ $\mathcal{M}\left(N_{6, n}\right)$ induced by the inclusion of $R$ in $N_{6, n}$ is injective, and we will treat $\mathcal{M}(R)$ as a subgroup of $\mathcal{M}\left(N_{6, n}\right)$.

Lemma 5.2 Suppose that $h: \mathcal{M}(R) \rightarrow \mathrm{GL}(2, \mathbb{C})$ is a homomorphism. Then, with respect to some basis, one of the following cases holds:

(a) $h\left(t_{\delta_{4}}\right)=h\left(t_{\delta_{5}}\right)=h\left(t_{\varepsilon_{2}}\right)=\lambda I, \quad \lambda \in\{-1,1\}$

(b) $h\left(t_{\delta_{4}}\right)=h\left(t_{\delta_{5}}\right)=h\left(t_{\varepsilon_{2}}\right)=\left(\begin{array}{cc}1 & 0 \\ 0 & -1\end{array}\right)$

(c) $h\left(t_{\delta_{5}}\right)=h\left(t_{\varepsilon_{2}}\right)=\left(\begin{array}{cc}1 & 1 \\ 0 & -1\end{array}\right), h\left(t_{\delta_{4}}\right)=\left(\begin{array}{cc}-1 & 0 \\ 1 & 1\end{array}\right)$

In particular, $h\left(t_{\delta_{4}}^{2}\right)=1$.

Proof For $i=4,5$, let $L_{i}=h\left(t_{\delta_{i}}\right), M=h\left(t_{\varepsilon_{2}}\right)$ and $U=h\left(u_{5}\right)$. Recall that the twists $t_{\delta_{i}}$ and $t_{\varepsilon_{2}}$ are pairwise conjugate, and each of them is conjugate to its inverse (by (R12)).

Case $1 M$ has only one eigenvalue $\lambda$ Since $M$ is conjugate to $M^{-1}$ we have $\lambda \in\{-1,1\}$. If $\operatorname{dim} E(M, \lambda)=2$, then we have case (a). Suppose that $\operatorname{dim} E(M, \lambda)=1$. Then with respect to some basis we have $M=\left(\begin{array}{ll}\lambda & 1 \\ 0 & \lambda\end{array}\right)$, and since $L_{5}$ commutes with $M, L_{5}=\left(\begin{array}{cc}\lambda & x \\ 0 & \lambda\end{array}\right)$ for some $x$.

If $E(M, \lambda) \neq E\left(L_{4}, \lambda\right)$, then we may arrange that the second vector of the basis is from $E\left(L_{4}, \lambda\right)$; thus, $L_{4}=\left(\begin{array}{ll}\lambda & 0 \\ y & \lambda\end{array}\right)$ for some $y$. From $M L_{4} M=L_{4} M L_{4}$ we obtain $y=-1$, and from $L_{4} L_{5} L_{4}=L_{5} L_{4} L_{5}$ we have $x=1$, hence $M=L_{5}$. By Lemma 3.4 (for $i=2, j=5$ ) we have $M^{2}=I$, which is a contradiction.

If $E(M, \lambda)=E\left(L_{4}, \lambda\right)$, then $L_{4}=\left(\begin{array}{cc}\lambda & y \\ 0 & \lambda\end{array}\right)$ for some $y$. From $M L_{4} M=L_{4} M L_{4}$ and $L_{4} L_{5} L_{4}=L_{5} L_{4} L_{5}$ we obtain $x=y=1$, hence $M=L_{5}$, which leads to a contradiction as above.

Case $2 M$ has two eigenvalues $\lambda, \mu$ With respect to some basis we have $M=$ $\left(\begin{array}{ll}\lambda & 0 \\ 0 & \mu\end{array}\right)$, and since $L_{5}$ and $U$ commute with $M$, they are also diagonal. In particular 
we have $U L_{5}=L_{5} U$ and $L_{5} U L_{5}=U$ (R12) gives $L_{5}^{2}=1$, which implies $\{\lambda, \mu\}=$ $\{-1,1\}$. Either $L_{5}=M$ or $L_{5}=-M$. In the latter case the braid relations $L_{5} L_{4} L_{5}=$ $L_{4} L_{5} L_{4}$ and $M L_{4} M=L_{4} M L_{4}$ imply $L_{4} M L_{4}=0$, a contradiction, hence $M=$ $L_{5}$.

If $E(M, 1) \neq E\left(L_{4}, 1\right)$, then with respect to some basis we have $M=\left(\begin{array}{cc}1 & 1 \\ 0 & -1\end{array}\right)$, $L_{4}=\left(\begin{array}{cc}-1 & 0 \\ x & 1\end{array}\right)$. From $M L_{4} M=L_{4} M L_{4}$ we have $x=1$ and we are in case (c). Analogously, if $E(M,-1) \neq E\left(L_{4},-1\right)$, then with respect to some basis we have $M=\left(\begin{array}{cc}-1 & 1 \\ 0 & 1\end{array}\right), L_{4}=\left(\begin{array}{cc}1 & 0 \\ 1 & -1\end{array}\right)$, and since $E(M, 1) \neq E\left(L_{4}, 1\right)$, we are in case (c) again. Finally, if $E(M, 1)=E\left(L_{4}, 1\right)$ and $E(M,-1)=E\left(L_{4},-1\right)$, then with respect to some basis we have $M=L_{4}=\left(\begin{array}{cc}1 & 0 \\ 0 & -1\end{array}\right)$ and we are in case (b).

Lemma 5.3 Suppose that $n \leq 1, f: \mathcal{M}\left(N_{6, n}\right) \rightarrow \mathrm{GL}(4, \mathbb{C})$ is a homomorphism and there exists a splitting $\mathbb{C}^{4}=V_{1} \oplus V_{2}$ such that $V_{i}$ is a 2-dimensional $\mathcal{M}(R)$-invariant subspace for $i=1,2$. Then $\operatorname{Im}(f)$ is abelian.

Proof Let $f^{\prime}$ be the restriction of $f$ to $\mathcal{M}(R)$. With respect to the splitting $\mathbb{C}^{4}=$ $V_{1} \oplus V_{2}$ we have $f^{\prime}=f_{1} \oplus f_{2}$ for some $f_{i}: \mathcal{M}(R) \rightarrow \mathrm{GL}(2, \mathbb{C}), i=1,2$. By Lemma 5.2 we have $f_{i}\left(t_{\delta_{4}}^{2}\right)=1$ for $i=1,2$, hence $f\left(t_{\delta_{4}}^{2}\right)=1$ and we are done by Lemma 5.1.

Lemma 5.4 Suppose that $n \leq 1, f: \mathcal{M}\left(N_{6, n}\right) \rightarrow \mathrm{GL}(4, \mathbb{C})$ is a homomorphism, $f\left(t_{\delta_{1}}\right)$ has only one eigenvalue and there exists a 2-dimensional $\mathcal{M}(R)$-invariant subspace. Then $\operatorname{Im} f$ is abelian.

Proof Fix a basis of $\mathbb{C}^{4}$ whose first two vectors span the $\mathcal{M}(R)$-invariant subspace. For $x \in \mathcal{M}(R)$ we have

$$
f(x)=\left(\begin{array}{cc}
h_{1}(x) & * \\
0 & h_{2}(x)
\end{array}\right)
$$

where $h_{1}(x)$ and $h_{2}(x)$ are 2-dimensional matrices. We apply Lemma 5.2 to the homomorphisms $h_{1}$ and $h_{2}$. Because $f\left(t_{\delta_{4}}\right)$ has only one eigenvalue, case (a) holds. It follows that $f\left(t_{\delta_{4}}\right)=\left(\begin{array}{cc}\lambda I & X \\ 0 & \lambda I\end{array}\right), f\left(t_{\delta_{5}}\right)=\left(\begin{array}{cc}\lambda I & Y \\ 0 & \lambda I\end{array}\right)$, for some $2 \times 2$ matrices $X, Y$ and $\lambda \in\{-1,1\}$. In particular $f\left(t_{\delta_{4}}\right)$ and $f\left(t_{\delta_{5}}\right)$ commute and we are done by Lemma 3.3.

Proof of Theorem 1.3 for $\boldsymbol{g}=\mathbf{6}, \boldsymbol{m}=\mathbf{4}$ Suppose that $n \in\{0,1\}$ and $f: \mathcal{M}\left(N_{6, n}\right) \rightarrow$ $\mathrm{GL}(4, \mathbb{C})$ is a homomorphism. For $1 \leq i \leq 5$, we set $L_{i}=f\left(t_{\delta_{i}}\right)$ and $M=f\left(t_{\varepsilon_{2}}\right)$, $U_{5}=f\left(u_{5}\right)$. We consider the following cases.

(1) $L_{1}$ has 4 eigenvalues.

(2) $L_{1}$ has 3 eigenvalues.

(3) $L_{1}$ has 2 eigenvalues with equal multiplicities. 
(4) $L_{1}$ has 2 eigenvalues with different multiplicities.

(5) $L_{1}$ has 1 eigenvalue.

In cases (1), (2), (3) it is easy to find a splitting $\mathbb{C}^{4}=V_{1} \oplus V_{2}$ such that $V_{i}$ is a 2-dimensional $\mathcal{M}(R)$-invariant subspace for $i=1$, 2. For example, suppose that $L_{1}$ has 3 eigenvalues $\lambda_{1}, \lambda_{2}, \lambda_{3}$ such that $\# \lambda_{1}=\# \lambda_{2}=1$ and $\# \lambda_{3}=2$. Then we take $V_{1}=E\left(L_{1}, \lambda_{1}\right) \oplus E\left(L_{1}, \lambda_{2}\right)$ and $V_{2}=E\left(L_{1}, \lambda_{3}\right)$ if $\operatorname{dim} E\left(L_{1}, \lambda_{3}\right)=2$ or $V_{2}=E^{2}\left(L_{1}, \lambda_{3}\right)$ if $\operatorname{dim} E\left(L_{1}, \lambda_{3}\right)=1$. Therefore in cases (1), (2), (3), we are done by Lemma 5.3.

Assume (5). Let $\lambda$ be the unique eigenvalue of $L_{1}$ and $k=\operatorname{dim} E\left(L_{1}, \lambda\right)$. If $k=4$ then $L_{1}=\lambda I$ and the image of $f$ is cyclic. If $k=2$ or $k=1$, then, respectively, $E\left(L_{1}, \lambda\right)$ or $E^{2}\left(L_{1}, \lambda\right)$ is a 2-dimensional $\mathcal{M}(R)$-invariant subspace, and we are done by Lemma 5.4. Suppose that $k=3$. If $E\left(L_{1}, \lambda\right) \neq E\left(L_{2}, \lambda\right)$ then $E\left(L_{1}, \lambda\right) \cap E\left(L_{2}, \lambda\right)$ is a 2-dimensional $\mathcal{M}(R)$-invariant subspace, and we are done by Lemma 5.4. If $E\left(L_{1}, \lambda\right)=E\left(L_{2}, \lambda\right)$, then with respect to some basis we have

$$
L_{1}=\left(\begin{array}{cccc}
\lambda & 0 & 0 & 0 \\
0 & \lambda & 0 & 0 \\
0 & 0 & \lambda & 1 \\
0 & 0 & 0 & \lambda
\end{array}\right), \quad L_{2}=\left(\begin{array}{cccc}
\lambda & 0 & 0 & x \\
0 & \lambda & 0 & y \\
0 & 0 & \lambda & z \\
0 & 0 & 0 & \lambda
\end{array}\right)
$$

In particular $L_{1}$ and $L_{2}$ commute and we are done by Lemma 3.3.

It remains to consider case (4). Suppose that $L_{1}$ has eigenvalues $\mu, \lambda$, with $\# \mu=1$ and $\# \lambda=3$. Since $L_{1}$ is conjugate to $L_{1}^{-1}$, we have $\{\mu, \lambda\}=\{-1,1\}$. It follows from Theorem 3.2 that there is a homomorphism $\tau\left(\mathcal{M}\left(N_{6}\right)\right) \rightarrow\{-1,1\}$ such that $\tau\left(t_{\delta_{1}}\right)=-1$. By multiplying $f$ by $\tau$ if necessary, we may assume $\mu=-1, \lambda=1$. The Jordan form of $L_{1}$ is one of the three matrices
(i) $\left(\begin{array}{rrrr}-1 & 0 & 0 & 0 \\ 0 & 1 & 0 & 0 \\ 0 & 0 & 1 & 0 \\ 0 & 0 & 0 & 1\end{array}\right)$,
(ii) $\left(\begin{array}{rrrr}-1 & 0 & 0 & 0 \\ 0 & 1 & 1 & 0 \\ 0 & 0 & 1 & 1 \\ 0 & 0 & 0 & 1\end{array}\right)$,
(iii) $\left(\begin{array}{rrrr}-1 & 0 & 0 & 0 \\ 0 & 1 & 0 & 0 \\ 0 & 0 & 1 & 1 \\ 0 & 0 & 0 & 1\end{array}\right)$.

In case (i) we have $L_{1}^{2}=I$ and we are done by Lemma 5.1.

In case (ii) the following subspaces are $\mathcal{M}(R)$-invariant: $E\left(L_{1},-1\right), E\left(L_{1}, 1\right)$, $E^{2}\left(L_{1}, 1\right), E^{3}\left(L_{1}, 1\right)$. It follows that

$$
M=\left(\begin{array}{cccc}
x_{1} & 0 & 0 & 0 \\
0 & x_{2} & v_{1} & v_{2} \\
0 & 0 & x_{3} & v_{3} \\
0 & 0 & 0 & x_{4}
\end{array}\right), \quad L_{4}=\left(\begin{array}{cccc}
y_{1} & 0 & 0 & 0 \\
0 & y_{2} & w_{1} & w_{2} \\
0 & 0 & y_{3} & w_{3} \\
0 & 0 & 0 & y_{4}
\end{array}\right)
$$


The braid relation $M L_{4} M=L_{4} M L_{4}$ implies $x_{i}=y_{i}$ for $1 \leq i \leq 4$. Since the first two vectors of the basis are eigenvectors of $M$, they have to correspond to different eigenvalues of $M$. Therefore $x_{2}=-x_{1}, x_{3}=x_{4}=1$ and $x_{1}=1$ or $x_{1}=-1$. In either case $M L_{4} M=L_{4} M L_{4}$ holds only if $M=L_{4}$. We are done by Lemma 3.3.

In case (iii) the following subspaces are $\mathcal{M}(R)$-invariant: $E\left(L_{1},-1\right), E\left(L_{1}, 1\right)$, $E^{2}\left(L_{1}, 1\right)$. We have $\operatorname{dim} E\left(L_{1}, 1\right)=2$. For $x \in \mathcal{M}(R)$ let $h(x)$ be the restriction of $f(x)$ to $E\left(L_{1}, 1\right)$. By applying Lemma 5.2 to $h$ we obtain three sub-cases.

Case (iii a) $h$ satisfies (a) of Lemma 5.2. We have

$$
M=\left(\begin{array}{rrrc}
-1 & 0 & 0 & 0 \\
0 & 1 & 0 & x_{1} \\
2 & 0 & 1 & x_{2} \\
0 & 0 & 0 & 1
\end{array}\right), \quad L_{4}=\left(\begin{array}{rrrc}
-1 & 0 & 0 & 0 \\
0 & 1 & 0 & y_{1} \\
0 & 0 & 1 & y_{2} \\
0 & 0 & 0 & 1
\end{array}\right)
$$

As in case (ii), the braid relation implies $M=L_{4}$ and we are done by Lemma 3.3.

Case (iii b) $h$ satisfies (b) of Lemma 5.2. By changing the basis of $E\left(L_{1}, 1\right)$, we may assume that

$$
M=\left(\begin{array}{rrrc}
1 & 0 & 0 & 0 \\
0 & -1 & 0 & x_{1} \\
0 & 0 & 1 & x_{2} \\
0 & 0 & 0 & 1
\end{array}\right), \quad L_{4}=\left(\begin{array}{rrrc}
1 & 0 & 0 & 0 \\
0 & -1 & 0 & y_{1} \\
0 & 0 & 1 & y_{2} \\
0 & 0 & 0 & 1
\end{array}\right)
$$

As in case (ii), the braid relation implies $M=L_{4}$ and we are done by Lemma 3.3.

Case (iii c) $h$ satisfies (c) of Lemma 5.2. By changing the basis of $E\left(L_{1}, 1\right)$ we may assume that

$M=\left(\begin{array}{rrrr}1 & 0 & 0 & 0 \\ 0 & 1 & 1 & x_{1} \\ 0 & 0 & -1 & x_{2} \\ 0 & 0 & 0 & 1\end{array}\right), \quad L_{4}=\left(\begin{array}{rrrr}1 & 0 & 0 & 0 \\ 0 & -1 & 0 & y_{1} \\ 0 & 1 & 1 & y_{2} \\ 0 & 0 & 0 & 1\end{array}\right), \quad L_{5}=\left(\begin{array}{rrrr}1 & 0 & 0 & 0 \\ 0 & 1 & 1 & z_{1} \\ 0 & 0 & -1 & z_{2} \\ 0 & 0 & 0 & 1\end{array}\right)$.

By solving the equations $M L_{4} M=L_{4} M L_{4}$ and $L_{5} L_{4} L_{5}=L_{4} L_{5} L_{4}$, we obtain $x_{2}=-\left(2 x_{1}+y_{1}+2 y_{2}\right), z_{2}=-\left(2 z_{1}+y_{1}+2 y_{2}\right)$, and from $M L_{5}=L_{5} M$ we obtain $x_{2}=z_{2}$. Thus $M=L_{5}$, and, by Lemma $3.4, L_{1}^{2}=1$. We are done by Lemma 5.1 .

\section{Homomorphisms between mapping class groups}

The aim of this section is to prove Theorem 1.4. Fix $g \geq 5$ and set $\mathcal{M}=\mathcal{M}\left(N_{g}\right)$. We are going to use the fact that $s=t_{\delta_{1}} \cdots t_{\delta_{g-1}}$ has finite order in $\mathcal{M}$ (equal to $g$ if it is 
even, or $2 g$ otherwise; see [23, Proposition 3.2]). By the relations (R1), (R4), we have

$$
t_{\delta_{i+1}} s=s t_{\delta_{i}} \quad \text { for } 1 \leq i \leq g-2 .
$$

By Theorem 3.2 we have $s \in[\mathcal{M}, \mathcal{M}]$ for $g \geq 7$ and $g=5, s^{2} \in[\mathcal{M}, \mathcal{M}]$ for $g=6$.

Proof of Theorem 1.4 Suppose that $g \geq 5, h<g$ and $f: \mathcal{M}\left(N_{g}\right) \rightarrow \mathcal{M}\left(N_{h}\right)$ is a homomorphism. Since $M\left(N_{h}\right)$ is abelian for $h \leq 2$, we are assuming $h \geq 3$.

Let $f^{\prime}: \mathcal{M}\left(N_{g}\right) \rightarrow \mathrm{GL}(h-1, \mathbb{C})$ be the composition $\Psi_{1} \circ f$ and $K=\operatorname{ker} \Psi_{1}$. By Theorem 1.3, $\operatorname{Im}\left(f^{\prime}\right)$ is abelian, hence $f\left(\left[\mathcal{M}\left(N_{g}\right), \mathcal{M}\left(N_{g}\right)\right]\right) \subseteq K$. Suppose that $g \geq 7$ or $g=5$. Then $f(s) \in K$, and since $K$ is torsion-free by Corollary 4.2, $f(s)=1$. This gives, by (2), $f\left(t_{\delta_{1}}\right)=f\left(t_{\delta_{2}}\right)$ and we are done by Lemma 3.3. If $g=6$ then $f\left(s^{2}\right) \in K$, which gives $f\left(s^{2}\right)=1$ and $f\left(t_{\delta_{2}}\right)=f\left(t_{\delta_{4}}\right)$. Since $t_{\delta_{1}}$ commutes with $t_{\delta_{4}}, f\left(t_{\delta_{1}}\right)$ commutes with $f\left(t_{\delta_{2}}\right)$ and we are done by Lemma 3.3.

Note that Theorems 1.3 and 1.4 are trivially true for $g \leq 3$ because $\operatorname{GL}(1, \mathbb{C})=\mathbb{C}^{*}$, $\mathcal{M}\left(N_{2}\right) \cong \mathbb{Z}_{2} \times \mathbb{Z}_{2}, M\left(N_{1}\right)=1$ are abelian groups. On the other hand, Corollary 6.2 below shows that both theorems are false for $g=4$ (recall that $\mathcal{M}\left(N_{3}\right) \cong \mathrm{GL}(2, \mathbb{Z})$ ). Let $D_{\infty}$ denote the infinite dihedral group, defined by the presentation

$$
D_{\infty}=\left\langle x, y \mid x^{2}=y^{2}=1\right\rangle .
$$

Lemma 6.1 There is an epimorphism $\phi: \mathcal{M}\left(N_{4}\right) \rightarrow D_{\infty}$.

Proof According to the main result of Szepietowski [27], simplified in [23], $\mathcal{M}\left(N_{4}\right)$ admits a presentation with generators $t_{\varepsilon_{2}}, t_{\delta_{i}}, u_{i}$ for $i=1,2,3$ and relations (R1), (R3), (R4), (R6), (R7), (R9), (R10), (R11), (R12) and

$$
\begin{gathered}
t_{\delta_{i+1}} u_{i} u_{i+1}=u_{i} u_{i+1} t_{\delta_{i}} \quad \text { for } i=1,2, \\
\left(t_{\varepsilon_{2}} u_{3}\right)^{2}=1, \quad t_{\delta_{1}}\left(t_{\delta_{2}} t_{\delta_{3}} u_{3} u_{2}\right) t_{\delta_{1}}=\left(t_{\delta_{2}} t_{\delta_{3}} u_{3} u_{2}\right) .
\end{gathered}
$$

It is easy to check that the mapping $\phi\left(t_{\varepsilon_{2}}\right)=x y, \phi\left(t_{\delta_{i}}\right)=1, \phi\left(u_{i}\right)=y$ for $i=1,2,3$ respects the defining relations of $\mathcal{M}\left(N_{4}\right)$, hence it defines a homomorphism onto $D_{\infty}$.

Corollary 6.2 For $h \geq 3$, there is a homomorphism $f: \mathcal{M}\left(N_{4}\right) \rightarrow \mathcal{M}\left(N_{h}\right)$ such that $\operatorname{Im}(f)$ is isomorphic to $D_{\infty}$.

Proof Fix $h \geq 3$. By the proof of Szepietowski [26, Theorem 3], $t_{\delta_{1}}$ can be written in $\mathcal{M}\left(N_{h}\right)$ as a product of two involutions $\sigma, \tau$. Since $t_{\delta_{1}}$ has infinite order in $\mathcal{M}\left(N_{h}\right)$, the mapping $x \mapsto \sigma, y \mapsto \tau$ defines an embedding $D_{\infty} \rightarrow \mathcal{M}\left(N_{h}\right)$. By precomposing this embedding with the epimorphism $\phi$ from Lemma 6.1, we obtain $f$. 
The following two theorems can be proved by the same method as Theorem 1.4. We leave the details to the reader.

Theorem 6.3 Suppose that $g \geq 5, g \geq 2 h+2$ and $f: \mathcal{M}\left(N_{g}\right) \rightarrow \mathcal{M}\left(S_{h}\right)$ is a homomorphism. Then $\operatorname{Im}(f)$ is abelian.

Theorem 6.4 Suppose that $g \geq 3$ and $h \leq 2 g$. Then the only homomorphism from $\mathcal{M}\left(S_{g}\right)$ to $\mathcal{M}\left(N_{h}\right)$ is the trivial one.

\section{Homomorphisms from $\mathcal{M}\left(N_{g}\right)$ to $\mathrm{GL}(g-1, \mathbb{C})$}

The aim of this section is to prove Theorem 1.5. The proof is divided into two cases, according to the parity of the genus.

Let $g=2 r+s, s \in\{1,2\}, S^{\prime}=S_{r, s}$ and $\iota: \mathcal{M}\left(S^{\prime}\right) \rightarrow \mathcal{M}\left(N_{g, n}\right)$ be the homomorphism from Corollary 3.6. If $f: \mathcal{M}\left(N_{g, n}\right) \rightarrow \operatorname{GL}(m, \mathbb{C})$ is a homomorphism, then we set $f^{\prime}=f \circ \iota$.

Proof of Theorem 1.5 for odd $\boldsymbol{g}$ Suppose that $N=N_{2 r+1}, r \geq 3$ and $f: \mathcal{M}(N) \rightarrow$ $\operatorname{GL}(2 r, \mathbb{C})$ is a homomorphism such that $\operatorname{Im}(f)$ is not abelian. By Theorem 1.2, $f^{\prime}$ is conjugate to the homological representation $\Phi$, and thus there exists a basis such that $f\left(t_{\varepsilon_{i}}\right)=f^{\prime}\left(t_{\alpha_{i}}\right)=A_{i}, f\left(t_{\delta_{2 i}}\right)=f^{\prime}\left(t_{\beta_{i}}\right)=B_{i}$ for $1 \leq i \leq r$ and $f\left(t_{\delta_{2 j+1}}\right)=$ $f^{\prime}\left(t_{\gamma_{j}}\right)=C_{j}$ for $1 \leq j \leq r-1$. Set $U_{k}=f\left(u_{k}\right)$ for $1 \leq k \leq 2 r$.

Since $U_{2 r}$ commutes with $A_{i}$ and $B_{i}$ for $1 \leq i \leq r$, and with $C_{j}$ for $j=1, \ldots, r-2$ (R6), (R8), by Lemma 2.1 we have

$$
U_{2 r}=\left(\begin{array}{cc}
\lambda I_{2 r-2} & 0 \\
0 & X
\end{array}\right)
$$

for some $2 \times 2$ matrix $X$. Since $U_{2 r}$ is conjugate to $U_{2 r}^{-1}$ we have $\lambda \in\{-1,1\}$ and by multiplying $f$ by $(-1)^{\mathrm{ab}}$ if necessary, we may assume $\lambda=1$. The relation $B_{r} U_{2 r} B_{r}=U_{2 r}$ (R12) implies

$$
X=\left(\begin{array}{rr}
x & 0 \\
y & -x
\end{array}\right)
$$

From (R11) and (R7), we have

$$
\begin{gathered}
U_{2 r-2}=\left(C_{r-1} B_{r} B_{r-1} C_{r-1}\right)^{-1} U_{2 r}\left(C_{r-1} B_{r} B_{r-1} C_{r-1}\right), \\
U_{2 r} U_{2 r-2}-U_{2 r-2} U_{2 r}=0,
\end{gathered}
$$


and since the left hand side of the last equation is equal to

$$
\left(1-x^{2}\right)\left(E_{2 r, 2 r-3}+E_{2 r-2,2 r-1}\right),
$$

where $E_{i, j}$ is the elementary matrix defined in Section $2, x^{2}=1$. We have $U_{2 r}^{-1}=U_{2 r}$, and from (R11) and (R9),

$$
\begin{aligned}
& U_{2 r-1}=\left(C_{r-1} B_{r}\right)^{-1} U_{2 r}\left(C_{r-1} B_{r}\right), \\
& U_{2 r} U_{2 r-1} U_{2 r}-U_{2 r-1} U_{2 r} U_{2 r-1}=0 .
\end{aligned}
$$

By considering the cases $x=1$ and $x=-1$ separately, we find that the left hand side of the last equation is of the form $(y-x)^{2} Z$, where $Z \neq 0$. Hence $x=y$ and $U_{2 r}=\Psi_{1}\left(u_{2 r}\right)$ if $x=1$, or $U_{2 r}=\Psi_{2}\left(u_{2 r}\right)$ if $x=-1$. By Theorem 3.1, $f$ is equal to $\Psi_{1}$ or $\Psi_{2}$ on the generators of $\mathcal{M}(N)$.

Now we will borrow some arguments from [16] to prove Lemma 7.3 below, which will be a starting point for the proof of Theorem 1.5 for even genus.

Lemma 7.1 Suppose that $n \leq 1, g \geq 5$ and $f: \mathcal{M}\left(N_{g, n}\right) \rightarrow \operatorname{GL}(m, \mathbb{C})$ is a homomorphism. If there is a flag $0=W_{0} \subset W_{1} \subset \cdots \subset W_{k}=\mathbb{C}^{m}$ of $\mathcal{M}\left(N_{g, n}\right)$-invariant subspaces such that $\operatorname{dim}\left(W_{i} / W_{i-1}\right)<g-1$ for $i=1, \ldots, k$, then $\operatorname{Im}(f)$ is abelian.

Proof We use a similar argument as in the proof of [16, Lemma 4.8]. For $i=1, \ldots, k$, set $m_{i}=\operatorname{dim}\left(W_{i} / W_{i-1}\right)$. Fix a basis $\left(v_{1}, \ldots, v_{m}\right)$ of $\mathbb{C}^{m}$, such that the vectors $v_{j}$ for $1 \leq j \leq m_{1}+\cdots+m_{i}$ form a basis of $W_{i}$. For $x \in \mathcal{M}\left(N_{g, n}\right)$, the matrix of $f(x)$ with respect to this basis is

$$
\left(\begin{array}{ccc}
X_{1} & * & * \\
0 & \ddots & * \\
0 & 0 & X_{k}
\end{array}\right),
$$

where $X_{i}$ is a square matrix of dimension $m_{i}$ for $i=1, \ldots, k$. Thus we have $k$ homomorphisms $f_{i}: \mathcal{M}\left(N_{g, n}\right) \rightarrow \mathrm{GL}\left(m_{i}, \mathbb{C}\right)$ defined by $f_{i}(x)=X_{i}$. Since $m_{i}<g-1$, the image of each $f_{i}$ is abelian by Theorem 1.3. It follows that $f\left[\mathcal{M}\left(N_{g, n}\right), \mathcal{M}\left(N_{g, n}\right)\right]$ is contained in the subgroup of upper triangular matrices with 1 on the diagonal. Since this subgroup is nilpotent and $\left[\mathcal{M}\left(S^{\prime}\right), \mathcal{M}\left(S^{\prime}\right)\right]$ is perfect [20, Theorem 4.2], it follows that $f^{\prime}\left[\mathcal{M}\left(S^{\prime}\right), \mathcal{M}\left(S^{\prime}\right)\right]$ is trivial, which means that $\operatorname{Im}\left(f^{\prime}\right)$ is abelian, hence so is $\operatorname{Im}(f)$.

Lemma 7.2 Suppose that $N=N_{2 r+2}, r \geq 3$ and $f: \mathcal{M}(N) \rightarrow \operatorname{GL}(2 r+1, \mathbb{C})$ is a homomorphism, such that $\operatorname{Im}(f)$ is not abelian. Then $L_{1}=f\left(t_{\delta_{1}}\right)$ has an eigenvalue $\lambda$ such that $\operatorname{dim} E\left(L_{1}, \lambda\right)=2 r$. 
Proof By [16, Corollary 4.6] applied to $f^{\prime}, L_{1}$ has at most two eigenvalues. It follows that there is an eigenvalue $\lambda$ with $\# \lambda \geq r+1 \geq 4$. Set $m=\operatorname{dim} E\left(L_{1}, \lambda\right)$. Since $\operatorname{Im}(f)$ is not abelian, $m \leq 2 r$. We are going to show that $m=2 r$.

Let $R$ be the subsurface obtained by removing from $N$ a regular neighbourhood of $\delta_{1} \cup \delta_{2}$. We have $R \approx N_{2 r, 1}$. We treat $\mathcal{M}(R)$ as a subgroup of $\mathcal{M}(N)$.

Suppose $m \leq 2 r-2$. Let $W=E^{k}\left(L_{1}, \lambda\right)$, where $k=\max \{4-m, 1\}$. Observe that $W$ is a $\mathcal{M}(R)$-invariant subspace with $3 \leq \operatorname{dim} W \leq 2 r-2$. By Lemma 7.1, $f(\mathcal{M}(R))$ is abelian, hence $f\left(t_{\delta_{4}}\right)$ and $f\left(t_{\delta_{5}}\right)$ commute. By Lemma 3.3, $\operatorname{Im}(f)$ is abelian, a contradiction.

Suppose that $m=2 r-1$ and set $L_{2}=f\left(t_{\delta_{2}}\right)$. If $E\left(L_{1}, \lambda\right) \neq E\left(L_{2}, \lambda\right)$, then $E\left(L_{1}, \lambda\right) \cap E\left(L_{2}, \lambda\right)$ is a $\mathcal{M}(R)$-invariant subspace of dimension $2 r-3$ or $2 r-2$ and we can use the same argument as above to obtain a contradiction. If $E\left(L_{1}, \lambda\right)=$ $E\left(L_{2}, \lambda\right)$, then by [16, Lemma 4.3] applied to $f^{\prime}, E\left(L_{1}, \lambda\right)$ is a $\mathcal{M}\left(S^{\prime}\right)$-invariant subspace of dimension $2 r-1$, and by [16, Lemma 4.8] $f^{\prime}$ is trivial. It follows that $\operatorname{Im} f$ is abelian, a contradiction.

Lemma 7.3 Suppose that $N=N_{2 r+2}, r \geq 3$ and $f: \mathcal{M}(N) \rightarrow \operatorname{GL}(2 r+1, \mathbb{C})$ is a homomorphism. If $r=3$ then assume that 1 is the unique eigenvalue of $f\left(t_{\delta_{1}}\right)$. Then either $\operatorname{Im}(f)$ is abelian, or with respect to some basis $f\left(t_{\varepsilon_{i}}\right)=A_{i}, f\left(t_{\delta_{2 i}}\right)=B_{i}$ for $i=1, \ldots, r$.

Proof Suppose that $\operatorname{Im}(f)$ is not abelian. The result will follow from [16, Lemma 4.7] applied to $f^{\prime}: \mathcal{M}\left(S^{\prime}\right) \rightarrow \operatorname{GL}(2 r+1, \mathbb{C})$. Therefore it suffices to show that $f^{\prime}$ satisfies the hypothesis of [16, Lemma 4.7], namely: (1) the Jordan form of $f^{\prime}\left(t_{\alpha_{1}}\right)$ is $\left(\begin{array}{cc}V & 0 \\ 0 & I_{2 r-1}\end{array}\right)$, and (2) $E\left(f^{\prime}\left(t_{\alpha_{1}}\right), 1\right) \neq E\left(f^{\prime}\left(t_{\beta_{1}}\right), 1\right)$.

By Lemma 7.2, $L_{1}=f^{\prime}\left(t_{\alpha_{1}}\right)=f\left(t_{\delta_{1}}\right)$ has an eigenvalue $\lambda$ with $\operatorname{dim} E\left(L_{1}, \lambda\right)=2 r$. If $r=3$, then $\lambda=1$ by assumption. For $r \geq 4$ we will prove $\lambda=1$ by using the argument from the proof of [16, Lemma 5.2]. Set $t_{1}=t_{\alpha_{1}}$ and choose 6 Dehn twits $t_{2}, \ldots, t_{7}$ about nonseparating simple closed curves on $S^{\prime}$ such that the lantern relation $t_{1} t_{2} t_{3} t_{4}=$ $t_{5} t_{6} t_{7}$ holds in $\mathcal{M}\left(S^{\prime}\right)$. By applying $f^{\prime}$ to both sides we obtain $L_{1} L_{2} L_{3} L_{4}=$ $L_{5} L_{6} L_{7}$, where $L_{i}=f^{\prime}\left(t_{i}\right)$. Since the $L_{i}$ are conjugate, we have $\operatorname{dim} E\left(L_{i}, \lambda\right)=2 r$ for $i=1, \ldots, 7$. Set $W=\bigcap_{i=1}^{7} E\left(L_{i}, \lambda\right)$ and observe that $\operatorname{dim} W>0$. For a nonzero vector $v \in W$, we have $\lambda^{4} v=L_{1} L_{2} L_{3} L_{4}(v)=L_{5} L_{6} L_{7}(v)=\lambda^{3} v$, hence $\lambda=1$. Since $\mathcal{M}\left(S^{\prime}\right)$ is perfect (Korkmaz [18]), det $L_{1}=1$ and $\lambda=1$ is the unique eigenvalue. It follows that $L_{1}$ has the desired Jordan form. 
Set $M=f^{\prime}\left(t_{\beta_{1}}\right)=f\left(t_{\delta_{2}}\right)$ and suppose $E\left(L_{1}, 1\right)=E(M, 1)$. Then $L_{1}$ and $M$ commute by the same argument as in case (5) of the proof of Theorem 1.3 for $(g, m)=(6,4)$. By Lemma 3.3, $\operatorname{Im}(f)$ is abelian, a contradiction. Thus $E\left(L_{1}, 1\right) \neq E(M, 1)$.

Proof of Theorem 1.5 for even $\boldsymbol{g}$ Suppose that $N=N_{2 r+2}, r \geq 4$, and

$$
f: \mathcal{M}(N) \rightarrow \mathrm{GL}(2 r+1, \mathbb{C})
$$

is a homomorphism such that $\operatorname{Im}(f)$ is not abelian. By Lemma 7.3, there is a basis such that $f\left(t_{\varepsilon_{i}}\right)=A_{i}$ and $f\left(t_{\delta_{2 i}}\right)=B_{i}$ for $1 \leq i \leq r$. Set $D_{i}=f\left(t_{\delta_{2 i+1}}\right)$ for $1 \leq i \leq r$ and $U_{j}=f\left(u_{j}\right)$ for $1 \leq j \leq 2 r+1$.

Fix $i \in\{1, \ldots, r-1\}$. For $j \notin\{i, i+1\}$ we have $D_{i} A_{j}=A_{j} D_{i}$ and $D_{i} B_{j}=B_{j} D_{i}$. Setting $M=D_{i}$ and $k=l=j$ in Lemma 2.1, we obtain

$$
D_{i}=\left(\begin{array}{ccc}
* & 0 & * \\
0 & \lambda I_{2} & 0 \\
* & 0 & *
\end{array}\right),
$$

where $\lambda$ is at the positions $(2 j-1,2 j-1)$ and $(2 j, 2 j)$. Since $D_{i}$ is conjugate to $A_{1}, 1$ is its unique eigenvalue, hence $\lambda=1$. It follows that $D_{i}$ has the form

$$
D_{i}=\left(\begin{array}{ccccc}
I_{2(i-1)} & 0 & 0 & 0 & 0 \\
0 & F_{11} & F_{12} & 0 & X_{1} \\
0 & F_{21} & F_{22} & 0 & X_{2} \\
0 & 0 & 0 & I_{2(r-i-1)} & 0 \\
0 & Y_{1} & Y_{2} & 0 & z
\end{array}\right),
$$

where $F_{k l}$ are $2 \times 2$ matrices, $X_{k}$ are $2 \times 1$ vectors, $Y_{k}$ are $1 \times 2$ vectors and $z$ is a complex number. The relations $D_{i} A_{i}=A_{i} D_{i}$ and $D_{i} A_{i+1}=A_{i+1} D_{i}$ give: $V F_{k k}=F_{k k} V, V X_{k}=X_{k}, Y_{k} V=Y_{k}$ for $k=1,2$, and $V F_{k l}=F_{k l}=F_{k l} V$ for $k \neq l$. It follows that

$$
\begin{array}{rlrl}
F_{11} & =\left(\begin{array}{cc}
s_{1} & t_{1} \\
0 & s_{1}
\end{array}\right), & F_{12}=\left(\begin{array}{cc}
0 & v_{1} \\
0 & 0
\end{array}\right), & X_{1}=\left(\begin{array}{c}
x_{1} \\
0
\end{array}\right), \\
F_{21}=\left(\begin{array}{cc}
0 & v_{2} \\
0 & 0
\end{array}\right), & F_{22}=\left(\begin{array}{cc}
s_{2} & t_{2} \\
0 & s_{2}
\end{array}\right), & X_{2}=\left(\begin{array}{c}
x_{2} \\
0
\end{array}\right), \\
Y_{1}=\left(\begin{array}{ll}
0 & y_{1}
\end{array}\right), & Y_{2}=\left(\begin{array}{ll}
0 & y_{2}
\end{array}\right) . &
\end{array}
$$

Since $s_{1}, s_{2}$ are eigenvalues, we have $s_{1}=s_{2}=1$ and det $D_{i}=z$, which gives $z=1$. Now, by solving the equations $B_{i} D_{i} B_{i}-D_{i} B_{i} D_{i}=0$ and $B_{i+1} D_{i} B_{i+1}-$ $D_{i} B_{i+1} D_{i}=0$, we obtain $t_{1}=t_{2}=1, v_{1} v_{2}=1, y_{2}=y_{1} v_{1}, x_{2}=x_{1} v_{2}, x_{1} y_{1}=0$. 
Thus, for $i=1, \ldots, r-1$ we have

$$
D_{i}=\left(\begin{array}{ccccccc}
I_{2(i-1)} & 0 & 0 & 0 & 0 & 0 & 0 \\
0 & 1 & 1 & 0 & \alpha_{i} & 0 & \alpha_{i} x_{i} \\
0 & 0 & 1 & 0 & 0 & 0 & 0 \\
0 & 0 & \alpha_{i}^{-1} & 1 & 1 & 0 & x_{i} \\
0 & 0 & 0 & 0 & 1 & 0 & 0 \\
0 & 0 & 0 & 0 & 0 & I_{2(r-i-1)} & 0 \\
0 & 0 & y_{i} & 0 & \alpha_{i} y_{i} & 0 & 1
\end{array}\right), \quad x_{i} y_{i}=0 .
$$

Analogous calculations, using the relations $A_{i} D_{r}=D_{r} A_{i}$ and $B_{i} D_{r}=D_{r} B_{i}$ for $1 \leq i \leq r, A_{r} D_{r}=D_{r} A_{r}$ and $D_{r} B_{r} D_{r}=B_{r} D_{r} B_{r}$, lead to the following form of $D_{r}$ :

$$
D_{r}=\left(\begin{array}{cccc}
I_{2 r-2} & 0 & 0 & 0 \\
0 & 1 & 1 & x_{r} \\
0 & 0 & 1 & 0 \\
0 & 0 & y_{r} & 1
\end{array}\right), \quad x_{r} y_{r}=0
$$

It is not possible that $x_{r}=y_{r}=0$, because then $D_{r}=A_{r}$ and Lemma 3.4 would give a contradiction. For $1 \leq i \leq r-1$, by solving the equation $D_{i} D_{r}-D_{r} D_{i}=0$ we obtain $x_{i} y_{r}=0$ and $x_{r} y_{i}=0$. It follows that either $x_{i}=0$ for all $i=1, \ldots, r$, or $y_{i}=0$ for all $i=1, \ldots, r$. We are going to show that it is possible to change the basis so that $\alpha_{i}=-1$ for $i=1, \ldots, r-1$ and $x_{r}+y_{r}=-2$. Suppose that the old basis is $\beta_{1}=\left(v_{1}, w_{1}, \ldots, v_{r}, w_{r}, v_{r+1}\right)$. We consider two cases.

Case $1 x_{r}=0$. Then $y_{r} \neq 0$ and the new basis is

$$
\begin{aligned}
v_{i}^{\prime} & =(-1)^{r-i} \alpha_{i} \cdots \alpha_{r-1} v_{i}, \quad w_{i}^{\prime}=(-1)^{r-i} \alpha_{i} \cdots \alpha_{r-1} w_{i}, \quad i=1, \ldots, r-1, \\
v_{r}^{\prime} & =v_{r}, \quad w_{r}^{\prime}=w_{r}, \quad v_{r+1}^{\prime}=-\frac{y_{r}}{2} v_{r+1} .
\end{aligned}
$$

In the new basis, we have $D_{r}=\Psi_{1}\left(t_{\delta_{2 r+1}}\right), D_{i}=C_{i}+x_{i}^{\prime}\left(E_{2 r+1,2 i}-E_{2 r+1,2 i+2}\right)$ for $i=1, \ldots, r-1$.

Case $2 y_{r}=0$. Then $x_{r} \neq 0$ and the new basis is

$$
\begin{aligned}
v_{i}^{\prime} & =(-1)^{r-i+1} \alpha_{i} \cdots \alpha_{r-1} \frac{x_{r}}{2} v_{i}, \quad w_{i}^{\prime}=(-1)^{r-i+1} \alpha_{i} \cdots \alpha_{r-1} \frac{x_{r}}{2} w_{i}, \\
i & =1, \ldots, r-1, \quad v_{r}^{\prime}=-\frac{x_{r}}{2} v_{r}, \quad w_{r}^{\prime}=-\frac{x_{r}}{2} w_{r}, \quad v_{r+1}^{\prime}=v_{r+1} .
\end{aligned}
$$

In the new basis we have: $D_{r}=\Psi_{2}\left(t_{\delta_{2 r+1}}\right), D_{i}=C_{i}+x_{i}^{\prime}\left(E_{2 i-1,2 r+1}-E_{2 i+1,2 r+1}\right)$ for $i=1, \ldots, r-1$. 
Since $U_{2 r+1}$ commutes with $A_{i}$ and $B_{i}$ for $1 \leq i \leq r-1$, by Lemma 2.1 we have

$$
U_{2 r+1}=\operatorname{diag}\left(\lambda_{1} I_{2}, \lambda_{2} I_{2}, \ldots, \lambda_{r-1} I_{2}, X\right)
$$

for some $3 \times 3$ matrix $X$. The relations $A_{r} U_{2 r+1}=U_{2 r+1} A_{r}(\mathrm{R} 8)$ and $D_{r} U_{2 r+1} D_{r}=$ $U_{2 r+1}$ (R12) imply that $X$ has the form

$$
X=\left(\begin{array}{ccc}
\lambda_{r} & \alpha & \lambda_{r} \\
0 & \lambda_{r} & 0 \\
0 & \beta & -\lambda_{r}
\end{array}\right) \quad \text { or } \quad X=\left(\begin{array}{ccc}
\lambda_{r} & \alpha & \beta \\
0 & \lambda_{r} & 0 \\
0 & \lambda_{r} & -\lambda_{r}
\end{array}\right),
$$

respectively, in Case 1 and Case 2. For $1 \leq i \leq r-1$, by the relation (R6) we have $D_{i} U_{2 r+1}-U_{2 r+1} D_{i}=0$. By solving this equation we obtain $\lambda_{i}=\lambda_{i+1}$ and $x_{i}^{\prime}=0$, hence $D_{i}=C_{i}$. We also see that $U_{2 r+1}$ has two eigenvalues $\lambda_{r},-\lambda_{r}$ with $\# \lambda_{r}=2 r$. Since $U_{2 r+1}$ is conjugate to $U_{2 r+1}^{-1}$, we have $\lambda_{r} \in\{-1,1\}$ and by multiplying $f$ by $(-1)^{\mathrm{ab}}$ if necessary, we may assume $\lambda_{r}=1$.

By the relation (R11), we have

$$
\begin{aligned}
U_{2 r} & =\left(B_{r} C_{r}\right)^{-1} U_{2 r+1}^{-1}\left(B_{r} C_{r}\right), \\
U_{2 r-1} & =\left(B_{r} C_{r} C_{r-1} B_{r}\right)^{-1} U_{2 r+1}\left(B_{r} C_{r} C_{r-1} B_{r}\right) .
\end{aligned}
$$

Similarly as in the proof for odd $g$, by solving $U_{2 r+1} U_{2 r-1}-U_{2 r-1} U_{2 r+1}=0$, we obtain $\beta=-2 \alpha$, and then by solving $U_{2 r+1} U_{2 r} U_{2 r+1}-U_{2 r} U_{2 r+1} U_{2 r}=0$, we obtain $\alpha=-1$ in Case 1, or $\alpha=1$ in Case 2. Hence $U_{2 r+1}=\Psi_{1}\left(u_{2 r+1}\right)$ in Case 1, or $U_{2 r+1}=\Psi_{2}\left(u_{2 r+1}\right)$ in Case 2. By Theorem 3.1, $f$ is equal to $\Psi_{1}$ in Case 1, and equal to $\Psi_{2}$ in Case 2, on generators of $\mathcal{M}(N)$.

\section{Homomorphisms from $\mathcal{M}\left(N_{8}\right)$ to $\operatorname{GL}(7, \mathbb{C})$}

The aim of this section is to prove Theorem 1.6. First we have to define the epimorphism $\epsilon: \mathcal{M}\left(N_{2 r+2}\right) \rightarrow \operatorname{Sp}\left(2 r, \mathbb{Z}_{2}\right)$.

Fix $r \geq 1$ and set $V=H_{1}\left(N_{2 r+2}, \mathbb{Z}_{2}\right) . V$ is a vector space over $\mathbb{Z}_{2}$ of dimension $2 r+2$ with basis $\overline{x_{i}}=\left[\xi_{i}\right]_{2}$ for $1 \leq i \leq 2 r+2$, where $\left[\xi_{i}\right]_{2}$ denotes the mod 2 homology class of the curve $\xi_{i}$. The mod 2 intersection pairing is the symmetric bilinear form on $V$ satisfying $\left\langle\overline{x_{i}}, \overline{x_{j}}\right\rangle=\delta_{i j}$. We define another basis for $V$. For $1 \leq i \leq r$ we set

$$
\begin{gathered}
v_{i}=\left[\varepsilon_{i}\right]_{2}=\overline{x_{1}}+\cdots+\overline{x_{2 i}}, \quad w_{i}=\left[\delta_{2 i}\right]_{2}=\overline{x_{2 i}}+\overline{x_{2 i+1}}, \\
c=\overline{x_{2 r+2}}, \quad d=\overline{x_{1}}+\cdots+\overline{x_{2 r+2}} .
\end{gathered}
$$

For $i, j \in\{1, \ldots, r\}$ we have $\left\langle v_{i}, v_{j}\right\rangle=\left\langle w_{i}, w_{j}\right\rangle=0,\left\langle v_{i}, w_{j}\right\rangle=\delta_{i j}$ and $\left\langle v_{i}, c\right\rangle=$ $\left\langle w_{i}, d\right\rangle=0$. We also have $\langle c, c\rangle=\langle c, d\rangle=1$ and $\langle d, d\rangle=0$. 
Lemma 8.1 Let Iso $(V)$ be the group of automorphisms of $V$ preserving the form $\langle\cdot, \cdot\rangle$. Then Iso( $V)$ is isomorphic to a semi-direct product $\operatorname{Sp}\left(2 r, \mathbb{Z}_{2}\right) \ltimes \mathbb{Z}_{2}^{2 r+1}$.

Proof Note that $d$ is the unique vector of $V$ such that $\left\langle\overline{x_{i}}, d\right\rangle=\left\langle\overline{x_{i}}, \overline{x_{i}}\right\rangle$ for $i=$ $1, \ldots, r$. It follows that $d$ is the unique vector satisfying $\langle x, d\rangle=\langle x, x\rangle$ for all $x \in V$, hence $d$ is fixed by all elements of $\operatorname{Iso}(V)$.

Let $W=\operatorname{span}\left\{v_{i}, w_{i} \mid i=1, \ldots, r\right\}$ and observe that the restriction of $\langle\cdot, \cdot\rangle$ to $W$ is nondegenerate and $\langle x, x\rangle=0$ for $x \in W$, hence it is a symplectic form on $W$. For $R \in \operatorname{Sp}(W)$, we define $A_{R} \in \operatorname{Iso}(V)$ as

$$
A_{R}(d)=d, \quad A_{R}(c)=c, \quad A_{R}(x)=R(x) \quad \text { for } x \in W .
$$

Any $x \in V$ can be written as $x=\gamma c+\delta d+w$, where $w \in W$ and $\gamma, \delta \in \mathbb{Z}_{2}$. We have $\langle x, c\rangle=\gamma+\delta$ and $\langle x, d\rangle=\gamma$. It follows that $W=\{x \in V \mid\langle x, d\rangle=\langle x, c\rangle=0\}$. Suppose $L \in \operatorname{Iso}(V)$ fixes $c$. Then, since $L(d)=d, L$ preserves $W$. Hence $L=A_{R}$ for some $R \in \mathrm{Sp}(W)$. It follows that the mapping $R \mapsto A_{R}$ defines an isomorphism $\mathrm{Sp}(W) \rightarrow \operatorname{Stab}_{\mathrm{Iso}(V)}(c)$.

For $x \in \mathbb{Z}_{2}$ and $z \in W$, we define $B_{x, z} \in \operatorname{Iso}(V)$ as

$$
B_{x, z}(d)=d, \quad B_{x, z}(c)=c+x d+z, \quad B_{x, z}(w)=w+\langle w, z\rangle d \quad \text { for } w \in W .
$$

For arbitrary $v \in V$, we have

$$
B_{x, z}(v)=v+\langle d, v\rangle z+\langle z+x d, v\rangle d .
$$

Set $N=\left\{B_{x, z} \mid x \in \mathbb{Z}_{2}, z \in W\right\}$. This is a subgroup of Iso( $\left.V\right)$ with the group law

$$
B_{x_{1}, z_{1}} B_{x_{2}, z_{2}}=B_{x_{1}+x_{2}+\left\langle z_{1}, z_{2}\right\rangle, z_{1}+z_{2}} .
$$

It follows that $N$ is abelian and $B_{x, z}^{2}=1$ for all $x, z$. Thus $N$ is isomorphic to $\mathbb{Z}_{2}^{2 r+1}$.

Let $L \in \operatorname{Iso}(V)$ be arbitrary. Since $\langle L(c), d\rangle=\langle L(c), L(d)\rangle=\langle c, d\rangle=1, L(c)=$ $c+x d+z$ for some $x \in \mathbb{Z}_{2}, z \in W$. It follows that $B_{x, z}^{-1} L \in \operatorname{Stab}_{\operatorname{Iso}(V)}(c)$ and hence $L=B_{x, z} A_{R}$ for some $R \in \mathrm{Sp}(W)$. This decomposition is clearly unique, and since $A_{R} B_{x, z} A_{R}^{-1}=B_{x, R(z)}, N$ is normal in $\operatorname{Iso}(V)$ and $\operatorname{Iso}(V)=N \rtimes \operatorname{Stab}_{\text {Iso( }(V)}(c)$.

Lemma 8.2 For $r \geq 2$, there is an epimorphism

$$
\epsilon: \mathcal{M}\left(N_{2 r+2}\right) \rightarrow \operatorname{Sp}\left(2 r, \mathbb{Z}_{2}\right),
$$

whose kernel is normally generated by $t_{\delta_{2 r+1}} u_{2 r+1}$ and $t_{\delta_{2 r+1}} t_{\varepsilon_{r}}^{-1}$. 
Proof Let $\mathcal{M}=\mathcal{M}\left(N_{2 r+2}\right)$. The action of $\mathcal{M}$ on $V=H_{1}\left(N_{2 r+2}, \mathbb{Z}_{2}\right)$ induces a homomorphism $\rho: \mathcal{M} \rightarrow \operatorname{Iso}(V)$, which was proved to be surjective in Gadgil and Pancholi [10], and McCarthy and Pinkall [22], and whose kernel is the normal closure of $t_{\delta_{2 r+1}} u_{2 r+1}$ by Szepietowski [29]. By Lemma 8.1, there exists a normal subgroup $N$ of $\operatorname{Iso}(V)$ such that $\operatorname{Iso}(V) / N$ is isomorphic to $\operatorname{Sp}\left(2 r, \mathbb{Z}_{2}\right)$. We define $\epsilon$ to be the composition of $\rho$ with the canonical projection $\operatorname{Iso}(V) \rightarrow \operatorname{Iso}(V) / N$.

Let $K$ be the normal closure of $t_{\delta_{2 r+1}} u_{2 r+1}$ and $t_{\delta_{2 r+1}} t_{\varepsilon_{r}}^{-1}$ in $\mathcal{M}$. We claim that $K \subseteq$ $\operatorname{ker} \epsilon$. We have $t_{\delta_{2 r+1}} u_{2 r+1} \in \operatorname{ker} \rho \subset \operatorname{ker} \epsilon$. Since $\left[\varepsilon_{r}\right]_{2}=v_{r}$ and $\left[\delta_{2 r+1}\right]_{2}=v_{r}+d$, for $x \in V$ we have $\rho\left(t_{\varepsilon_{r}}\right)(x)=x+\left\langle v_{r}, x\right\rangle v_{r}$ and $\rho\left(t_{\delta_{2 r+1}}\right)(x)=x+\left\langle v_{r}+d, x\right\rangle\left(v_{r}+d\right)=$ $B_{1, v_{r}}\left(\rho\left(t_{\varepsilon_{r}}\right)(x)\right)$. Thus $\rho\left(t_{\delta_{2 r+1}} t_{\varepsilon_{r}}^{-1}\right)=B_{1, v_{r}} \in N$ and $t_{\delta_{2 r+1}} t_{\varepsilon_{r}}^{-1} \in \operatorname{ker} \epsilon$. It follows that there is an induced epimorphism

$$
\epsilon^{\prime}: \mathcal{M} / K \rightarrow \operatorname{Iso}(V) / N \cong \operatorname{Sp}\left(2 r, \mathbb{Z}_{2}\right) .
$$

To prove that $\epsilon^{\prime}$ is an isomorphism, it suffices to show $[\mathcal{M}: K] \leq\left|\operatorname{Sp}\left(2 r, \mathbb{Z}_{2}\right)\right|$. We are going to prove the last inequality by exhibiting an epimorphism $\operatorname{Sp}\left(2 r, \mathbb{Z}_{2}\right) \rightarrow \mathcal{M} / K$.

Observe that the map $\eta: \mathcal{M}\left(S^{\prime}\right) \rightarrow \mathcal{M} / K$ defined to be the composition of $\iota: \mathcal{M}\left(S^{\prime}\right) \rightarrow$ $\mathcal{M}$ from Corollary 3.6 with the canonical projection $\pi: \mathcal{M} \rightarrow \mathcal{M} / K$ is surjective because $\mathcal{M}$ is generated by twists about curves on $P\left(S^{\prime}\right)$ and $t_{\delta_{2 r+1}} u_{2 r+1}$ by Theorem 3.1. Gluing a disc along the boundary component of $S^{\prime}$ bounding a pair of pants with $\alpha_{r}$ and $\gamma_{r}$ induces an epimorphism $\mathcal{M}\left(S^{\prime}\right) \rightarrow \mathcal{M}\left(S_{r, 1}\right)$ whose kernel is normally generated by $t_{\gamma_{r}} t_{\alpha_{r}}^{-1}$ (see [16, Proposition 3.8]). Since $\iota\left(t_{\gamma_{r}} t_{\alpha_{r}}^{-1}\right)=t_{\delta_{2 r+1}} t_{\varepsilon_{r}}^{-1} \in K$, it follows that we have an induced epimorphism $\eta^{\prime}: \mathcal{M}\left(S_{r, 1}\right) \rightarrow \mathcal{M} / K$. There is an epimorphism $\mathcal{M}\left(S_{r, 1}\right) \rightarrow \operatorname{Sp}\left(2 r, \mathbb{Z}_{2}\right)$ induced by the action of $\mathcal{M}\left(S_{r, 1}\right)$ on $H_{1}\left(S_{r, 1}, \mathbb{Z}_{2}\right)$ whose kernel is normally generated by $t_{\alpha_{1}}^{2}$ (see Berrick, Gebhardt and Paris [2, Theorem 5.7]; here we are using the assumption $r \geq 2$ ). By applying Lemma 3.4 (with $i=r, j=2 r+1)$ to $\pi: \mathcal{M} \rightarrow \mathcal{M} / K$, we have $\eta^{\prime}\left(t_{\alpha_{1}}^{2}\right)=\pi\left(t_{\delta_{1}}^{2}\right)=1$. It follows that there is an induced epimorphism $\eta^{\prime \prime}: \operatorname{Sp}\left(2 r, \mathbb{Z}_{2}\right) \rightarrow \mathcal{M} / K$.

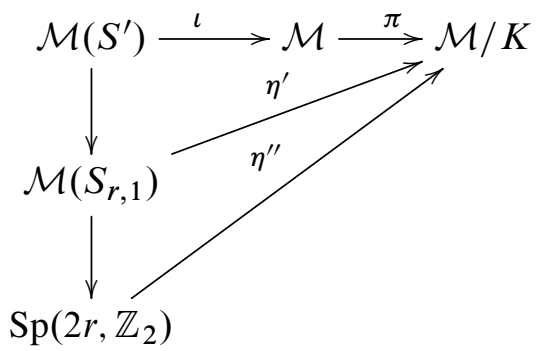

The existence of $\eta^{\prime \prime}$ proves that $\epsilon^{\prime}$ is an isomorphism and $K=\operatorname{ker} \epsilon$. 
Lemma 8.3 Suppose that $f: \mathcal{M}\left(N_{8}\right) \rightarrow \operatorname{GL}(7, \mathbb{C})$ is a homomorphism such that $f\left(t_{\delta_{1}}\right)$ has order 2 . Then $f$ or $(-1)^{\mathrm{ab}} f$ factors through the epimorphism $\epsilon: \mathcal{M}\left(N_{8}\right) \rightarrow$ $\operatorname{Sp}\left(6, \mathbb{Z}_{2}\right)$.

Proof Let $H$ be the normal closure of $t_{\delta_{1}}^{2}$ in $\mathcal{M}=\mathcal{M}\left(N_{8}\right)$ and $G=\mathcal{M} / H$. Since $H \subseteq \operatorname{ker} f$, we have a homomorphism $f^{\prime}: G \rightarrow \operatorname{GL}(7, \mathbb{C})$ such that $f=f^{\prime} \circ \pi$, where $\pi: \mathcal{M} \rightarrow G$ is the canonical projection. There is a homomorphism $\rho: \mathfrak{S}_{8} \rightarrow G$, defined as $\rho\left(\sigma_{i}\right)=\pi\left(t_{\delta_{i}}\right)$, where $\sigma_{i}=(i, i+1)$, for $1 \leq i \leq 7$. Let $\phi: \mathfrak{S}_{8} \rightarrow \operatorname{GL}(7, \mathbb{C})$ be the composition $\phi=f^{\prime} \circ \rho$. If $\phi$ is reducible, then $\operatorname{Im}(\phi)$ is abelian by Lemma 2.2, $f\left(t_{\delta_{1}}\right)=\phi\left(\sigma_{1}\right)=\phi\left(\sigma_{2}\right)=f\left(t_{\delta_{2}}\right)$, and $\operatorname{Im}(f)$ is also abelian by Lemma 3.3, which implies $f\left(t_{\delta_{1}}\right)=1$ by Theorem 3.2, a contradiction. Hence $\phi$ is irreducible and since det $f\left(t_{\delta_{1}}\right)=1$ (by Theorem 3.2), $\phi$ is the tensor product of the standard and sign representations (by Lemma 2.2). For $1 \leq i \leq 7$, set $L_{i}=f\left(t_{\delta_{i}}\right)=\phi\left(\sigma_{i}\right)$. With respect to some basis $\left(v_{1}, \ldots, v_{7}\right)$, we have

$$
L_{1}=\operatorname{diag}\left(A,-I_{5}\right), \quad L_{7}=\operatorname{diag}\left(-I_{5}, B\right), \quad L_{i}=\operatorname{diag}\left(-I_{i-2}, C,-I_{6-i}\right)
$$

for $2 \leq i \leq 6$, where

$$
A=\left(\begin{array}{ll}
1 & -1 \\
0 & -1
\end{array}\right), \quad B=\left(\begin{array}{ll}
-1 & 0 \\
-1 & 1
\end{array}\right), \quad C=\left(\begin{array}{rrr}
-1 & 0 & 0 \\
-1 & 1 & -1 \\
0 & 0 & -1
\end{array}\right) .
$$

Let $M$ be the matrix of $f\left(\varepsilon_{3}\right)$. Since $M$ commutes with $L_{i}$ for $i \neq 6$ (R5), it preserves $E\left(L_{i}, 1\right)=\operatorname{span}\left\{v_{i}\right\}$. Hence $M\left(v_{i}\right)=x_{i} v_{i}$ for $i \neq 6$ and $M\left(v_{6}\right)=y_{1} v_{1}+\cdots+y_{7} v_{7}$ for some complex numbers $x_{i}, y_{j}$. By solving the equations $M L_{i}=L_{i} M$ for $1 \leq i \leq 5$ and $i=7$, we obtain

$$
x_{i}=x_{1}, \quad y_{i}=i y_{1} \quad \text { for } 1 \leq i \leq 5, \quad y_{6}=x_{1}+6 y_{1}, \quad x_{7}=y_{6}-2 y_{7} .
$$

Since $M$ and $L_{i}$ are conjugate, they have the same eigenvalues, which gives $x_{1}=-1$ and $y_{6}=-x_{7}$. If $y_{6}=1$, then $y_{1}=1 / 3$ and $y_{7}=1$, which contradicts the braid relation $M L_{6} M=L_{6} M L_{6}$ (R5). Hence $y_{6}=-1, y_{1}=0$ and $y_{7}=-1$, which means $M=L_{7}$.

For $i=1, \ldots, 7$, let $U_{i}$ be the matrix of $f\left(u_{i}\right)$. Since $U_{7}$ commutes with $L_{j}$ for $1 \leq j \leq 5$ (R6) and with $M=L_{7}$ (R8), we obtain, as above, that

$$
\begin{aligned}
& U_{7}\left(v_{i}\right)=x v_{i} \quad \text { for } 1 \leq i \leq 5, \\
& U_{7}\left(v_{6}\right)=y\left(v_{1}+2 v_{2}+3 v_{3}+4 v_{4}+5 v_{5}\right)+(x+6 y) v_{6}+z v_{7}, \\
& U_{7}\left(v_{7}\right)=(x+6 y-2 z) v_{7},
\end{aligned}
$$


for some complex numbers $x, y, z$. Since $U_{7}$ is conjugate to its inverse, and $x$ is an eigenvalue of multiplicity at least 5 , we have $x= \pm 1$, and by multiplying $f$ by $(-1)^{\mathrm{ab}}$ if necessary, we may assume $x=-1$. By (R11) we have $U_{5}=$ $\left(L_{6} L_{7} L_{5} L_{6}\right)^{-1} U_{7}\left(L_{6} L_{7} L_{5} L_{6}\right)$, and by solving $U_{5} U_{7}=U_{7} U_{5}$ we obtain $y=0$. Since $\operatorname{det} U_{7}= \pm 1$, either $-1-2 z=1$ or $-1-2 z=-1$. In the latter case we have $U_{7}=-I$, and since $U_{6}$ is conjugate to $U_{7}$, we have $U_{6}=-I$, and the relation $L_{6} U_{7} U_{6}=U_{7} U_{6} L_{7}$ (R10) gives $L_{6}=L_{7}$, a contradiction. Hence $z=-1$ and $U_{7}=L_{7}$.

We have $M=U_{7}=L_{7}$, and since $L_{7}^{2}=I$, we have $\left\{t_{\delta_{7}} t_{\varepsilon_{3}}^{-1}, t_{\delta_{7}} u_{7}\right\} \subset$ ker $f$, which implies, by Lemma 8.2, that $f$ factors through $\epsilon$.

Proof of Theorem 1.6 Suppose that $f: \mathcal{M}\left(N_{8}\right) \rightarrow \operatorname{GL}(7, \mathbb{C})$ is a homomorphism, such that $\operatorname{Im}(f)$ is not abelian. By Lemma 7.2, $L=f\left(t_{\delta_{1}}\right)$ has an eigenvalue $\lambda$ such that $\operatorname{dim} E(L, \lambda)=6$. Since $L$ is conjugate to $L^{-1}$, we have $\lambda^{2}=1$. Suppose that $\lambda=-1$. Then since $\operatorname{det} L=1$ we have $\# \lambda=6$, and there is another eigenvalue $\mu=1$. It follows that $L$ has order 2 and the case (2) holds by Lemma 8.3. If $\lambda=1$ then it must be the unique eigenvalue, and the case (3) holds by Lemma 7.3 and the proof of Theorem 1.5 for even $g$.

Remark 8.4 Suppose that $G$ is a finite quotient of $\mathcal{M}\left(N_{g}\right)$ for $g \geq 7, g \neq 8$, and $f: G \rightarrow \mathrm{GL}(g-1, \mathbb{C})$ is a homomorphism. Then, by Theorem $1.5, \operatorname{Im}(f)$ is abelian. For example, by Lemma 8.2, for $r \geq 4$ the image of every homomorphism $\operatorname{Sp}\left(2 r, \mathbb{Z}_{2}\right) \rightarrow \operatorname{GL}(2 r+1, \mathbb{C})$ is abelian. It is a classical result that $\operatorname{Sp}\left(2 r, \mathbb{Z}_{d}\right)$ is perfect for $r \geq 3$ and all $d$ (note that the last group is a quotient of $\mathcal{M}\left(S_{r}\right)$, which is perfect for $r \geq 3$ [24]). It follows that for $r \geq 4$ the only homomorphism from $\operatorname{Sp}\left(2 r, \mathbb{Z}_{2}\right)$ to $\mathrm{GL}(2 r+1, \mathbb{C})$ is the trivial one.

\section{Acknowledgements}

I wish to thank the referee for helpful suggestions. This work was supported by NCN grant number 2012/05/B/ST1/02171.

\section{References}

[1] J Aramayona, J Souto, Homomorphisms between mapping class groups, Geom. Topol. 16 (2012) 2285-2341 MR3033518

[2] J A Berrick, V Gebhardt, L Paris, Finite index subgroups of mapping class groups, to appear in Proc. London Math. Soc 
[3] J S Birman, D R J Chillingworth, On the homeotopy group of a non-orientable surface, Proc. Cambridge Philos. Soc. 71 (1972) 437-448 MR0300288

[4] J Bray, S Linton, S Norton, R Parker, S Rogers, I Suleiman, J Tripp, P Walsh, RA Wilson, Atlas of finite group representations (2001) Available at http:// brauer.maths.qmul.ac.uk/Atlas/v3/

[5] F Castel, Geometric representations of the braid groups arXiv:1104.3698

[6] D R J Chillingworth, A finite set of generators for the homeotopy group of a nonorientable surface, Proc. Cambridge Philos. Soc. 65 (1969) 409-430 MR0235583

[7] D B A Epstein, Curves on 2-manifolds and isotopies, Acta Math. 115 (1966) 83-107 MR0214087

[8] J Franks, M Handel, Triviality of some representations of $\operatorname{MCG}\left(S_{g}\right)$ in $\operatorname{GL}(n, \mathbb{C})$, $\operatorname{Diff}\left(S^{2}\right)$ and Homeo( $\left.\mathbb{T}^{2}\right)$, Proc. AMS 141 (2013) 2951-2962 MR3068948

[9] W Fulton, J Harris, Representation theory: A first course, Graduate Texts in Mathematics 129, Springer, New York (1991) MR1153249

[10] S Gadgil, D Pancholi, Homeomorphisms and the homology of non-orientable surfaces, Proc. Indian Acad. Sci. Math. Sci. 115 (2005) 251-257 MR2161731

[11] P A Gastesi, A note of the Torelli spaces of non-orientable compact Klein surfaces, Ann. Acad. Sci. Fenn. Math. 24 (1999) 23-30 MR1677997

[12] F J González-Acuña, J M Márquez-Bobadilla, On the homeotopy group of the non orientable surface of genus three, Rev. Colombiana Mat. 40 (2006) 75-79 MR2321592

[13] W J Harvey, M Korkmaz, Homomorphisms from mapping class groups, Bull. London Math. Soc. 37 (2005) 275-284 MR2119027

[14] A Hatcher, Algebraic topology, Cambridge Univ. Press (2002) MR1867354

[15] M Korkmaz, Low-dimensional linear representations of mapping class groups arXiv: 1104.4816

[16] M Korkmaz, The symplectic representation of the mapping class group is unique arXiv: 1108.3241

[17] M Korkmaz, First homology group of mapping class groups of nonorientable surfaces, Math. Proc. Cambridge Philos. Soc. 123 (1998) 487-499 MR1607985

[18] M Korkmaz, Low-dimensional homology groups of mapping class groups: A survey, Turkish J. Math. 26 (2002) 101-114 MR1892804

[19] M Korkmaz, Problems on homomorphisms of mapping class groups, from: "Problems on mapping class groups and related topics", (B Farb, editor), Proc. Sympos. Pure Math. 74, Amer. Math. Soc. (2006) 81-89 MR2264533

[20] M Korkmaz, J D McCarthy, Surface mapping class groups are ultrahopfian, Math. Proc. Cambridge Philos. Soc. 129 (2000) 35-53 MR1757776 
[21] W B R Lickorish, Homeomorphisms of non-orientable two-manifolds, Proc. Cambridge Philos. Soc. 59 (1963) 307-317 MR0145498

[22] J D McCarthy, U Pinkall, Representing homology automorphisms of nonorientable surfaces, preprint MPI/SFB 85-11, Max Planck Institute (2004) Available at http:// www. math.msu.edu/ mccarthy

[23] L Paris, B Szepietowski, A presentation for the mapping class group of a nonorientable surface arXiv:1308.5856

[24] J Powell, Two theorems on the mapping class group of a surface, Proc. Amer. Math. Soc. 68 (1978) 347-350 MR0494115

[25] M Stukow, Generating mapping class groups of nonorientable surfaces with boundary, Adv. Geom. 10 (2010) 249-273 MR2629814

[26] B Szepietowski, Mapping class group of a non-orientable surface and moduli space of Klein surfaces, C. R. Math. Acad. Sci. Paris 335 (2002) 1053-1056 MR1955587

[27] B Szepietowski, A presentation for the mapping class group of the closed nonorientable surface of genus 4, J. Pure Appl. Algebra 213 (2009) 2001-2016 MR2533302

[28] B Szepietowski, Embedding the braid group in mapping class groups, Publ. Mat. 54 (2010) 359-368 MR2675928

[29] B Szepietowski, Crosscap slides and the level 2 mapping class group of a nonorientable surface, Geom. Dedicata 160 (2012) 169-183 MR2970047

Institute of Mathematics, Gdańsk University

Wita Stwosza 57, 80-952 Gdańsk, Poland

blaszep@mat .ug.edu.pl

Received: 6 May 2013 Revised: 17 January 2014 